\title{
Sharp-interface model for eutectic alloys Part I: Concentration dependent surface tension
}

\author{
W. DREYER ${ }^{\dagger}$ AND B. WAGNER ${ }^{\ddagger}$ \\ Weierstrass Institute for Applied Analysis and Stochastics, \\ Mohrenstrasse 39, D-10117 Berlin, Germany
}

[Received 24 March 1004 and in revised form 10 February 2005]

\begin{abstract}
We consider the problem of phase separation in eutectic alloy such as e.g. $S n P b$. For this we derive a phase field model from an atomistic point of view. We find the surface energy to be anisotropic, having in general a nonlinear dependence on concentration. We use matched asymptotic analysis to obtain a corresponding sharp-interface model. The resulting expression for the surface tension agrees with that found on the basis of classical thermodynamics for jump conditions at singular interfaces. A boundary integral formulation of the sharp-interface model enables us to numerically describe the motion and deformation of the binary alloy.
\end{abstract}

\section{Introduction}

Already in 1958 J. W. Cahn and J. E. Hilliard [4] considered the possibility of anisotropic surface tension in a crystal lattice. This is generically the case when modeling phase separation in multiphase systems such as binary alloys. A number of models have been developed generalizing the Cahn-Hilliard equation to multi-component systems by introducing a vector-valued order parameter and by making some general assumptions on the form of the gradient energy [6], [28], [26]. Using matched asymptotic expansions, corresponding sharp-interface models were then developed and expressions for surface tension were determined on the basis of results by Herring on anisotropic surface energy [13], [20].

Recently, a phase field model for the description and simulation of coarsening processes occurring in binary alloys, that are caused by diffusion in local inhomogeneous stress fields, has been formulated in [9]. There, the model is applied to the eutectic solder alloy consisting of lead and tin. Figure 1 shows a typical morphology that developed from an initially fine mix of alternating layers of lead-rich and tin-rich regions after 20 hours of slow cooling. The regions are resolved on a $\mu \mathrm{m}$-scale. This coarsening process is initiated by diffusion subjected to the effects of anisotropic surface tension and of thermomechanical stress fields. Here, the symmetry of the crystal lattices in the two phases is tetragonal and face-centered-cubic in the lead-rich and tin-rich phase, respectively. For further related work see also e.g. [27], [17], [11].

We consider coarsening processes that may be subjected to external thermomechanical loads. The given temperature $T$ is assumed to be uniform in space. In this case the morphology and its

\footnotetext{
†-mail: dreyer@wias-berlin.de

‡E-mail: wagnerb@wias-berlin.de
} 


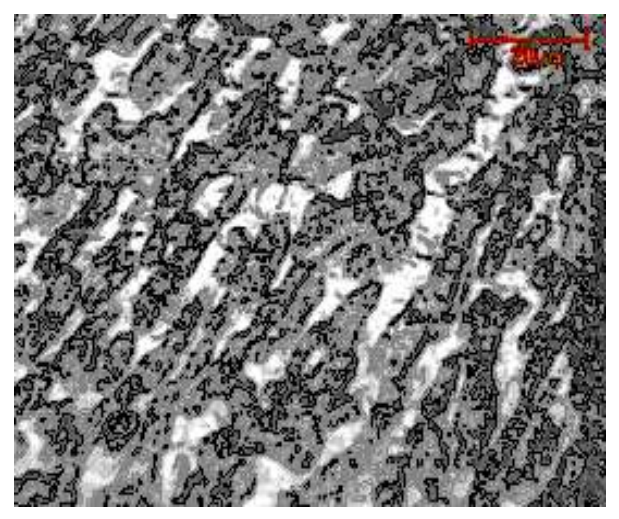

FIG. 1. Lead rich (dark) lamellae

evolution is described by the fields

$$
\begin{aligned}
& v_{i}(t, \boldsymbol{X})-\text { displacement, leading to the strain } \varepsilon_{i j}=\frac{1}{2}\left(\frac{\partial v_{i}}{\partial X_{j}}+\frac{\partial v_{j}}{\partial X_{i}}\right), \\
& c(t, X)-\text { mass concentration (e.g. tin). }
\end{aligned}
$$

Here $t$ denotes the time and $\boldsymbol{X}=\left(X_{1}, X_{2}, X_{3}\right)$ are the Lagrange coordinates with respect to a Cartesian frame of reference of the material particles of the alloy, which are the smallest volume units that can be resolved on the space scale considered. The motion of a particle with coordinates $\boldsymbol{X}$ is given by the function $\boldsymbol{x}=\chi(t, \boldsymbol{X})$, which gives the actual coordinates $\boldsymbol{x}=\left(x_{1}, x_{2}, x_{3}\right)$ of the particle at time $t$. Its displacement is $v_{i}=\chi_{i}-X_{i}$. The objective of the phase field model is the determination of the fields (1.1). Sometimes it is useful to refer the fields to the actual coordinates. This can be done by the definitions

$$
\tilde{c}(t, \boldsymbol{x})=c\left(t, \chi^{-1}(t, \boldsymbol{x})\right) \quad \text { and } \quad \tilde{v}_{i}(t, \boldsymbol{x})=v_{i}\left(t, \chi^{-1}(t, \boldsymbol{x})\right) .
$$

The field equations for the displacement and the concentration rely on the quasistatic momentum balance and on the conservation law for the content of one of the two constituents of the binary alloy.

In Part I of this study we assume that the displacement field is given, so that we only need the conservation law

$$
\rho_{0} \frac{\partial c}{\partial t}+\frac{\partial J_{k}}{\partial X_{k}}=0 .
$$

Here and throughout, we use the Einstein summation convention. The constant $\rho_{0}$ denotes the mass density of the reference state, which is here given by a homogeneous phase mixture at the eutectic composition, and $J_{k}$ are the components of the diffusion flux.

The conservation law (1.3) becomes a field equation for the concentration if we relate the diffusion flux to the concentration by a constitutive law that we determine as follows. In the appendix of [9], Dreyer and Müller have exploited the second law of thermodynamics relying on the assumption that the specific free energy, $\psi$, is given by a function of the type

$$
\psi=\hat{\psi}\left(T, c, \frac{\partial c}{\partial X_{i}}, \frac{\partial^{2} c}{\partial X_{i} \partial X_{j}}, \varepsilon_{i j}\right),
$$


and they choose Joule/kg as the unit of $\psi$. In Section 2 of this paper we will give a short survey on the main points of the derivation. Furthermore Dreyer and Müller showed in [9] that in accordance with the second law of thermodynamics, the diffusion flux $J_{k}$ may be related to the specific free energy by the constitutive law

$$
J_{k}=-\frac{B_{k l}}{T} \frac{\partial \mu}{\partial X_{l}} \quad \text { with } \quad \mu=\frac{\partial \psi}{\partial c}-\frac{\partial}{\partial X_{m}}\left(\frac{\partial \psi}{\partial\left(\partial c / \partial X_{m}\right)}\right)+\frac{\partial^{2}}{\partial X_{m} \partial X_{n}}\left(\frac{\partial \psi}{\partial\left(\partial^{2} c /\left(\partial X_{m} \partial X_{n}\right)\right)}\right),
$$

which generalizes the well known diffusion law according to Fick. The newly introduced quantities $B_{k l}$ are the components of the mobility matrix, which can be related to the matrix of diffusion coefficients.

The idea we pursue in this article is to form the free energy density $\psi$ of the two-phase mixture by interpolation within the interfacial region of the two phases, and use this for the derivation of the diffusion flux for the binary alloy. The resulting model shows that when mechanical effects are neglected the coefficients of the surface tension terms introduce some anisotropy which is due to the concentration dependence of the coefficients. This effect is the main focus of Part I of this study. Hence, here the only field equation will be the diffusion equation. In Part II we will discuss the contributions of mechanical effects to anisotropy.

The main objectives of Part I of this study on the coarsening problem of eutectic alloys are the following:

(i) Atomistic motivations and derivations of the phase field model, which describes anisotropic surface tension and mechanical stresses that appear during coarsening processes.

(ii) Establishment of the corresponding sharp-interface limit and its numerical exploitation for a strongly reduced model that exclusively takes care of anisotropic surface tension and ignores mechanical effects.

For this reason, a comparison of numerical experiments with real life processes in eutectic alloys will be postponed to Part II of this study.

In the next section we begin with a presentation of the Helmholtz free energy, composed of the potential energy between particles and the entropic part and derive expressions for the surface tension coefficients. The corresponding coefficients in the expression for the diffusion flux are then found via the mean field limit. In Section 3 we use matched asymptotic analysis to derive the corresponding sharp-interface model. Interestingly, the expression for the surface tension that results for the sharp-interface limit allows only two-fold symmetry if mechanical effects are neglected. In Section 4 it is shown how the same expression can be found from classical thermodynamics arguments based on the derivation of jump conditions at free boundaries. Finally, in Section 5 we derive a boundary integral formulation for the sharp-interface model that enables us to employ the nonstiff numerical method, due to [16], for our numerical solution to the problem.

\section{Atomistic modeling of phase field systems}

\subsection{The free energy function for the phase mixture}

In this section we establish the constitutive law for the free energy density from an atomistic point of view. To this end we consider separately the two phases, called $\alpha$-phase and $\beta$-phase, of the twophase mixture and calculate first their individual specific free energy densities $\psi_{\alpha}$ and $\psi_{\beta}$. In the 
second step we form the free energy density of the two-phase mixture by interpolation within the interfacial region according to

$$
\psi=u \psi_{\alpha}+(1-u) \psi_{\beta}, \quad \text { with } \quad u= \begin{cases}1 & \text { for } \boldsymbol{X} \in \alpha \text {-phase } \\ 0 & \text { for } \boldsymbol{X} \in \beta \text {-phase }\end{cases}
$$

where $u$ is the scaled concentration indicating the phase that occupies the location $\boldsymbol{X}$.

We may relate $u$ to the concentration field by

$$
u(t, \boldsymbol{X})=\frac{c^{\beta}(T)-c(t, \boldsymbol{X})}{c^{\beta}(T)-c^{\alpha}(T)}
$$

where $c^{\alpha}(T)$ and $c^{\beta}(T)$ refer to the equilibrium concentration of the $\alpha$-phase and $\beta$-phase, respectively. Thus the scaled concentration variable $u$ may change continuously in the interfacial region from 0 to 1 . It remains to derive the free energy functions $\psi_{\alpha}$ and $\psi_{\beta}$ for the individual phases.

\subsection{The free energy functions for the individual phases $\alpha$ and $\beta$}

We consider a body that consists exclusively of the pure phase $\gamma$, where $\gamma$ may generically represent either phase $\alpha$ or $\beta$. The body consists of a crystal lattice, whose symmetry is given, and the lattice sites are randomly occupied by $A$-type and $B$-type atoms. We decompose the total free energy of the body into its energetic and entropic parts and write

$$
\Psi_{\gamma}=U_{\gamma}-T S_{\gamma} .
$$

$U_{\gamma}$ and $S_{\gamma}$ denote the internal energy and the entropy of the body, which will now be determined successively.

The internal energy can be decomposed into a thermal part, $U_{\gamma}(T)$, which does not interest us at this point, and the potential part, $U_{\text {pot }}$, which is due to the interaction energy between all particles. For simplicity, we assume central forces to act between the atoms $a$ and $b,\{a, b\} \in\{1, \ldots, N\}$, and we write

$$
U_{\gamma \mid \text { pot }}=\Phi_{\gamma}\left(x_{i}^{1}, \ldots, x_{i}^{N}\right)=\frac{1}{2} \sum_{\substack{a, b=1 \\ a \neq b}}^{N} \varphi_{\gamma}^{a b}\left(r^{a b}\right) \quad \text { with } \quad r^{a b}=\left|x^{b}-x^{a}\right|
$$

where the $3 N$-tuple $\left(x_{i}^{1}, \ldots, x_{i}^{N}\right)$ contains the current positions of the atoms. While central force potentials are best suited to explore the key ideas and the atomistic origin of the various contributions appearing in the diffusion flux of phase field models, we note that they are in general not appropriate to describe the behavior of crystal lattices and may lead to some unrealistic results such as the socalled Cauchy paradox [18]. In [10] some of the resulting shortcomings are discussed.

We introduce the microscopic displacements

$$
\xi_{i}^{a}=x_{i}^{a}-X_{i}^{a}
$$

in order to substitute the current positions by the Lagrange positions of the atoms. These substitutions take care of terms up to second order in the displacements, resulting in a linear theory of elasticity. 
The case $T=0$. In this subsection we establish the expansion of $\Phi_{\gamma}$ at $T=0$. In our derivation we introduce the effects of thermal expansion and other eigenstrains only when taking the mean field limit, which simplifies the calculation considerably. The justification for this procedure is given in [18]. First, from 2.4 we obtain

$$
\begin{aligned}
U_{\gamma \mid \mathrm{pot}} & =\Phi_{\gamma}\left(x_{i}^{1}, \ldots, x_{i}^{N}\right)=\Phi_{\gamma}\left(X_{i}^{1}+\xi_{i}^{1}, \ldots, X_{i}^{N}+\xi_{i}^{N}\right) \\
& =\Phi_{\gamma}\left(X_{i}^{1}, \ldots, X_{i}^{N}\right)+\frac{1}{2} \sum_{\substack{a, b=1 \\
a \neq b}}^{N} \frac{\partial^{2} \Phi_{\gamma}\left(X_{i}^{1}, \ldots, X_{i}^{N}\right)}{\partial X_{k}^{a} \partial X_{l}^{b}} \xi_{k}^{a} \xi_{l}^{b} .
\end{aligned}
$$

The first derivative does not appear here because at $T=0$ the potential energy assumes its minimum. Next, we note that there are three different potential functions, viz. $\varphi_{\gamma}^{A A}$ for $A A$ interactions, $\varphi_{\gamma}^{B B}$ for $B B$-interactions and $\varphi_{\gamma}^{A B}$ for $A B$-interactions. The introduction of the particle concentration operator

$$
\hat{y}(a)= \begin{cases}0 & \text { if } a \text { indicates an } A \text {-type atom, } \\ 1 & \text { if } a \text { indicates an } B \text {-type atom, }\end{cases}
$$

permits us to represent a generic potential $\varphi_{\gamma}^{a b}$ by

$$
\begin{aligned}
\varphi_{\gamma}^{a b}= & (1-\hat{y}(a))(1-\hat{y}(b)) \varphi_{\gamma}^{A A}+\hat{y}(a) \hat{y}(b) \varphi_{\gamma}^{B B} \\
& +[\hat{y}(b)(1-\hat{y}(a))+\hat{y}(a)(1-\hat{y}(b))] \varphi_{\gamma}^{A B} .
\end{aligned}
$$

This representation will be introduced now in both terms of 2.6. As a consequence, there appear new quantities, which are defined as

$$
\begin{aligned}
& \varphi_{\gamma}\left(\Delta^{a b}\right)=\varphi_{\gamma}^{A B}\left(\Delta^{a b}\right)-\frac{1}{2}\left(\varphi_{\gamma}^{A A}\left(\Delta^{a b}\right)+\varphi_{\gamma}^{A B}\left(\Delta^{a b}\right)\right), \\
& \tilde{\varphi}_{\gamma}\left(\Delta^{a b}\right)=\frac{1}{2}\left(\varphi_{\gamma}^{B B}\left(\Delta^{a b}\right)-\varphi_{\gamma}^{A A}\left(\Delta^{a b}\right)\right),
\end{aligned}
$$

where $\Delta^{a b}$ denotes the magnitude of the reference distance between atoms $a$ and $b$. The term $\Phi_{\gamma}\left(X_{i}^{1}, \ldots, X_{i}^{N}\right)$ in 2.6 can now be rewritten as

$$
\begin{aligned}
\Phi_{\gamma}\left(X_{i}^{1}, \ldots, X_{i}^{N}\right)=\frac{1}{2} \sum_{\substack{a, b=1 \\
a \neq b}}^{N} \varphi_{\gamma}^{a b}\left(\Delta^{a b}\right) \\
\quad=\sum_{\substack{a, b=1 \\
a \neq b}}^{N}\left(\frac{1}{2} \varphi_{\gamma}^{A A}\left(\Delta^{a b}\right)+\hat{y}(a)(1-\hat{y}(b)) \varphi_{\gamma}\left(\Delta^{a b}\right)+\frac{1}{2}(\hat{y}(a)+\hat{y}(b)) \tilde{\varphi}_{\gamma}\left(\Delta^{a b}\right),\right.
\end{aligned}
$$

and the sum in 2.6 results in a similar manner.

Next we carry out the mean field limit, where quantities that describe the state of an individual atom are replaced by their respective mean values, which are assumed to vary slowly in time and space. A detailed discussion on various aspects of the mean field limit is found in [12], [18] and [8]. Regarding the atomistic quantities appearing in this paper we define the mean field by the following rules: 
(i) substitute the particle operator, which can only assume the values 0 or 1 , by the particle concentration, which may change continuously between 0 and 1 :

$$
\begin{aligned}
& \hat{y}(a) \rightarrow y\left(t, X^{a}\right) \equiv y(t, \boldsymbol{X}), \\
& \hat{y}(b) \rightarrow y\left(t, X^{a}+\Delta^{a b}\right) \approx y(t, \boldsymbol{X})+\frac{\partial y(t, \boldsymbol{X})}{\partial X_{k}} \Delta_{k}^{a b}+\frac{\partial^{2} y(t, \boldsymbol{X})}{\partial X_{k} \partial X_{l}} \Delta_{k}^{a b} \Delta_{l}^{a b},
\end{aligned}
$$

(ii) substitute the atomic displacement by the mean displacement:

$$
\begin{aligned}
& \xi_{i}^{a} \rightarrow u_{i}\left(t, X^{a}\right) \equiv u_{i}(t, \boldsymbol{X}), \\
& \xi_{i}^{b} \rightarrow u_{i}\left(t, X^{a}+\Delta^{a b}\right) \approx u_{i}(t, \boldsymbol{X})+\frac{\partial u_{i}(t, \boldsymbol{X})}{\partial X_{k}} \Delta_{k}^{a b} .
\end{aligned}
$$

Note that the coordinate $X^{b}$ denotes the location of atom $b$. It is related to an expansion for atom $a$ at the coordinate $X^{a}$, which in turn is abbreviated by $X$.

Finally, we collect all terms appearing and obtain three different contributions to the potential part of the total free energy of the pure phase $\gamma \in\{\alpha, \beta\}$ :

$$
\begin{aligned}
U_{\gamma \mid \mathrm{pot}}= & \sum_{a}\left[\psi_{\gamma}^{A A}+\psi_{\gamma}^{\tilde{\varphi}} y+\psi_{\gamma}^{\varphi} y(1-y)\right. \\
& -\left(a_{\gamma \mid k l}^{\varphi} y-\frac{1}{2} a_{\gamma \mid k l}^{\tilde{\varphi}}+\left(A_{\gamma \mid k l m n o p}^{\varphi} y-\frac{1}{2} A_{\gamma \mid k l m n o p}^{\tilde{\varphi}}\right) \varepsilon_{m n} \varepsilon_{o p}\right) \frac{\partial^{2} y}{\partial X_{k} \partial X_{l}} \\
& \left.+\frac{1}{2}\left(A_{\gamma \mid \text { mnop }}^{A A}+A_{\gamma \mid \text { mnop }}^{\tilde{\varphi}} y+A_{\gamma \mid \text { mnop }}^{\varphi}\right) \varepsilon_{m n} \varepsilon_{o p}\right]
\end{aligned}
$$

Here, the dependence on the concentration and the strain is explicit and the newly introduced coefficients are constants which can be calculated from the given interaction potentials. Their definitions are given in appendix B. The first line of 2.13 gives the classical local contribution and its third term is the energy of mixing. The first two terms of the second line describe the nonlocal interactions. Those terms depend on the concentration and are related to the classical Cahn-Hilliard model (see [4]). Note that the first derivatives of the atomic concentration, $\partial y / \partial X_{k}$, do not appear in the representation 2.13, which is due to our restriction to crystal lattices which have either tetragonal or cubic symmetry. Finally, the third line gives the purely elastic part of the potential energy, and the bracket in front of $\varepsilon_{m n} \varepsilon_{o p}$ is the stiffness matrix, which, however, turns out to depend on concentration.

The case $T>0$. For $T>0$ a competition of the energy of mixing and the entropy arises. In a phase $\gamma$ with a disordered distribution of the $A$-type and $B$-type atoms over the lattice sites, the entropy $S_{\gamma}$ is given by

$$
S_{\gamma}=-k \sum_{a}[y \log (y)+(1-y) \log (1-y)]
$$

Here $k$ is the Boltzmann constant. If the coefficient $\psi_{\gamma}^{\varphi}$ in 2.13 is positive, the energy of mixing and the entropy may combine so that the local part of the free energy becomes a nonconvex function.

Next, we discuss further effects which are induced for $T>0$. These are eigenstresses as a consequence of eigenstrains, and the most prominent representative is the eigenstrain due to thermal 
expansion. Other eigenstrains are due to point defects, dislocations and misfit strain. The latter arises for example during phase transitions if the new phase needs more space than the old one. All these effects are described by eigenstrains that have the generic form

$$
\varepsilon_{\gamma \mid m n}^{*}=\varepsilon_{\gamma \mid m n}^{*}(T, c) .
$$

Eigenstrains can be incorporated into the model, i.e. into the equation (2.13), by the substitution

$$
\varepsilon_{m n} \rightarrow\left(\varepsilon_{m n}-\varepsilon_{\gamma \mid m n}^{*}\right) .
$$

Suppressing its thermal part, we obtain the total free energy for a pure phase $\gamma$ :

$$
\begin{aligned}
\Psi_{\gamma}= & \sum_{a}\left[\psi_{\gamma}^{A A}+\psi_{\gamma}^{\tilde{\varphi}} y+\psi_{\gamma}^{\varphi} y(1-y)\right. \\
& -\left(a_{\gamma \mid k l}^{\varphi} y-\frac{1}{2} a_{\gamma \mid k l}^{\tilde{\varphi}}+\left(A_{\gamma \mid k l m n o p}^{\varphi} y-\frac{1}{2} A_{\gamma \mid k l m n o p}^{\tilde{\varphi}}\right) b\left(\varepsilon_{m n}-\varepsilon_{\gamma \mid m n}^{*}\right)\left(\varepsilon_{o p}-\varepsilon_{\gamma \mid o p}^{*}\right)\right) \frac{\partial^{2} y}{\partial X_{k} \partial X_{l}} \\
& +\frac{1}{2}\left(A_{\gamma \mid m n o p}^{A A}+A_{\gamma \mid m n o p}^{\tilde{\varphi}} y+A_{\gamma \mid m n o p}^{\varphi}\right)\left(\varepsilon_{m n}-\varepsilon_{\gamma \mid m n}^{*}\right)\left(\varepsilon_{o p}-\varepsilon_{\gamma \mid o p}^{*}\right) \\
& \left.+k T \sum_{a}(y \log (y)+(1-y) \log (1-y))\right] .
\end{aligned}
$$

Recall that we need to know the free energy as a function of the mass concentration $c$ rather than a function of the particle concentration $y$. Both quantities are related by the equation

$$
y=\frac{M_{A} c}{M_{B}-\left(M_{B}-M_{A}\right) c},
$$

where $M_{A}$ and $M_{B}$ are the molecular weights of the constituents $A$ and $B$, respectively.

\subsection{The specific free energy and the diffusion flux of the phase mixture}

From 2.17) we may read off the specific free energy $\psi_{\gamma}$ of the pure generic phase $\gamma$. We consider one mole, and abbreviate each term of the sum in 2.17 by $\tilde{\psi}_{\gamma \mid a}$, with $\psi_{\gamma}=\left[N_{A} / M(c)\right] \tilde{\psi}_{\gamma \mid a}$, where $N_{A}=6.023 \times 10^{23}$ particles/mole is the Avogadro number. We recall the interpolation 2.1] and obtain the specific free energy of the phase mixture, viz.

$$
\psi=\frac{N_{A}}{M(c)}\left(u(c) \tilde{\psi}_{\alpha \mid a}+(1-u(c)) \tilde{\psi}_{\beta \mid a}\right),
$$

where

$$
M(c)=\frac{M_{A} M_{B}}{M_{B}-\left(M_{B}-M_{A}\right) c}
$$

is the mean molecular weight of the binary mixture. A function of the type

$$
\psi=\psi_{0}\left(c, \varepsilon_{r s}\right)-a_{j l}\left(c, \varepsilon_{r s}\right) \frac{\partial^{2} c}{\partial X_{j} \partial X_{l}}+b_{j l}\left(c, \varepsilon_{r s}\right) \frac{\partial c}{\partial X_{j}} \frac{\partial c}{\partial X_{l}}
$$


is obtained, where the identification of the local part of the specific free energy $\psi_{0}$ and the matrix functions $a_{j l}$ and $b_{j l}$ is done after carrying out the necessary differentiations in order to transfer the $y$-dependent functions $\tilde{\psi}_{\alpha \mid a}$ and $\tilde{\psi}_{\beta \mid a}$ into functions of the mass concentration $c$. This calculation is easy but lengthy and left to the interested reader.

Finally, we use the constitutive law 2.1 to calculate the diffusion flux. We abbreviate $A_{j l}=$ $a_{j l}+b_{j l}$ and obtain an expression of the following type:

$$
\begin{aligned}
J_{k}= & -\frac{B_{k i}}{T} \frac{\partial}{\partial X_{i}}\left(\frac{\partial \psi_{0}\left(c, \varepsilon_{r s}\right)}{\partial c}-2 A_{j l}\left(c, \varepsilon_{r s}\right) \frac{\partial^{2} c}{\partial X_{j} \partial X_{l}}-\frac{\partial A_{j l}\left(c, \varepsilon_{r s}\right)}{\partial c} \frac{\partial c}{\partial X_{j}} \frac{\partial c}{\partial X_{l}}\right. \\
& \left.-2 \frac{\partial A_{j l}\left(c, \varepsilon_{r s}\right)}{\partial \varepsilon_{m n}} \frac{\partial c}{\partial X_{j}} \frac{\partial \varepsilon_{m n}}{\partial X_{l}}-\frac{\partial^{2} a_{j l}\left(c, \varepsilon_{r s}\right)}{\partial \varepsilon_{m n} \partial \varepsilon_{o p}} \frac{\partial \varepsilon_{o p}}{\partial X_{j}} \frac{\partial \varepsilon_{m n}}{\partial X_{l}}-\frac{\partial a_{j l}\left(c, \varepsilon_{r s}\right)}{\partial \varepsilon_{m n}} \frac{\partial^{2} \varepsilon_{m n}}{\partial X_{j} \partial X_{l}}\right) .
\end{aligned}
$$

\section{Sharp-interface limit}

In the following we ignore mechanical contributions to the free energy and to the diffusion flux, and we consider exclusively the effect of a simple anisotropy of the gradient coefficients $A_{k l}$, which become functions of the concentration.

A detailed evaluation of the mechanical phenomena is the content of Part II of this study. For the case we consider here, the diffusion flux $(2.22)$ reduces to

$$
J_{k}=-\frac{B_{k i}}{T} \frac{\partial}{\partial X_{i}}\left(\frac{\partial \psi_{0}(c)}{\partial c}-2 A_{j l}(c) \frac{\partial^{2} c}{\partial X_{j} \partial X_{l}}-\frac{\partial A_{j l}(c)}{\partial c} \frac{\partial c}{\partial X_{j}} \frac{\partial c}{\partial X_{l}}\right)
$$

For simplicity, we assume now a constant and isotropic mobility tensor $B_{k i}=B \delta_{k i}$, and for further simplification we refer to an eutectic alloy. We restrict ourselves to the regime below the eutectic line of the $S n P b$ phase diagram, which gives the coexistence region of a binary phase mixture, with regions of high lead ( $\alpha$-phase) and high tin ( $\beta$-phase) concentration. The lattice symmetry of the $\alpha$-phase is cubic, whereas the $\beta$-phase exhibit tetragonal symmetry. This symmetry leads to the gradient coefficients

$$
A_{j l}^{\alpha}=a^{\alpha} \delta_{j l}, \quad A_{j l}^{\beta}=\left(\begin{array}{ccc}
a_{1}^{\beta} & 0 & 0 \\
0 & a_{1}^{\beta} & 0 \\
0 & 0 & a_{2}^{\beta}
\end{array}\right),
$$

with constants $a^{\alpha}, a_{1}^{\beta}$ and $a_{2}^{\beta}$ for the $\alpha$ - and $\beta$-phase, respectively [9]. The interface region of the two phases is the only region with significant contributions to the higher gradients. Here we interpolate as in 2.1], i.e.

$$
A_{j l}=A_{j l}^{\alpha} u+A_{j l}^{\beta}(1-u)
$$

with $u$ defined as in 2.2. Note that since we neglect all mechanical effects the Lagrange coordinates coincide with the actual coordinates $\boldsymbol{x}$. We now substitute the resulting expression for the diffusion flux into the conservation equation 1.3 and transform the equation to the scaled concentration variable $u(t, \boldsymbol{x})$, which is 0 in the $\beta$-phase and 1 in the $\alpha$-phase. We nondimensionalize via

$$
x_{i}=L \tilde{x}_{i}, \quad t=\omega \tilde{t}, \quad \psi=\bar{\psi} F(u),
$$

and obtain, after dropping the tildes for the governing equation,

$$
\frac{\partial u}{\partial t}=\Delta \mu
$$




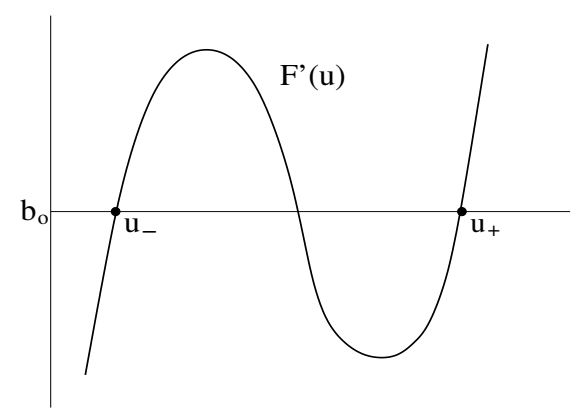

FIG. 2 .

where $\triangle$ denotes the Laplacian operator and

$$
\mu=F^{\prime}(u)-\varepsilon^{2}\left(2 A_{k l}(u) \frac{\partial^{2} u}{\partial x_{k} \partial x_{l}}+A_{k l}^{\prime}(u) \frac{\partial u}{\partial x_{k}} \frac{\partial u}{\partial x_{l}}\right)
$$

in a domain $\Omega=\Omega_{+} \cup \Omega_{-}$with

$$
\varepsilon^{2}=\frac{B \omega}{\bar{T} L^{2}} \quad \text { and } \quad \omega=\frac{\bar{T}\left(c_{\beta}-c_{\alpha}\right)^{2}}{B \bar{\psi}}
$$

where $\bar{T}$ is the constant temperature. The gradient energy coefficients $A_{k l}(u)$ are in general nonlinear functions of $u(t, \boldsymbol{x})$, where we abbreviated $A_{k l}(c(u))$ by $A_{k l}(u)$ with $c(u)=c^{\beta}-$ $\left(c^{\beta}-c^{\alpha}\right) u(t, \boldsymbol{x})$. The prime denotes the derivative with respect to $u$. The free energy $F(u)$ has the form of a double-well potential (see Fig. 2). On the boundary $\partial \Omega$ we take zero flux and the variational condition

$$
\boldsymbol{n} \cdot \nabla \mu=0, \quad \boldsymbol{n} \cdot \nabla u=0 .
$$

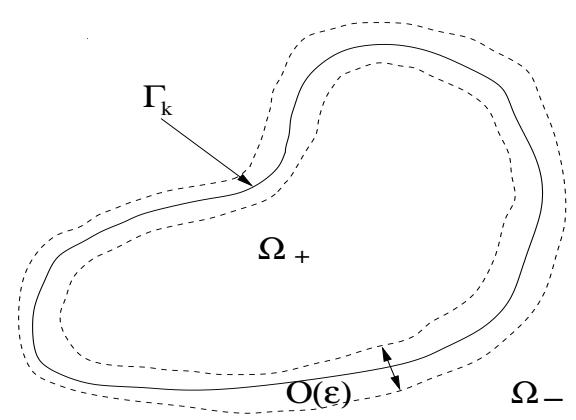

FIG. 3. Sketch of sharp interface.

Solutions of this problem reach phase equilibrium after $O(1)$ time. Near phase equilibrium, a solution has developed an internal boundary layer structure, having a width of $O(\varepsilon)$ and approaching sharp interfaces $\Gamma_{i}$ of the appearing precipitates as $\varepsilon \rightarrow 0$ (see Fig. 3). The dynamics of the 
precipitates evolves then on the slow time scale $\tau=\varepsilon t$ and the governing equation describing this is

$$
\varepsilon \frac{\partial u}{\partial \tau}=\Delta \mu
$$

where $\mu$ is given by 3.5 .

The solution to the corresponding internal boundary layer problem will yield the boundary condition for the "outer problem", i.e. in the region of $\Omega$ outside that boundary layer.

\subsection{Outer problem}

Let $u$ have the asymptotic expansion

$$
u(\tau, \boldsymbol{x} ; \varepsilon)=u_{\mathrm{o}}(\tau, \boldsymbol{x})+\varepsilon u_{1}(\tau, \boldsymbol{x})+\varepsilon^{2} u_{2}(\tau, \boldsymbol{x})+O\left(\varepsilon^{3}\right) .
$$

Correspondingly we can develop $\mu$ as

$$
\mu(\tau, \boldsymbol{x} ; \varepsilon)=\mu_{\mathrm{o}}(\tau, \boldsymbol{x})+\varepsilon \mu_{1}(\tau, \boldsymbol{x})+\varepsilon^{2} \mu_{2}(\tau, \boldsymbol{x})+O\left(\varepsilon^{3}\right) .
$$

Substituting into 3.4 we obtain together with 3.5 for the leading order problem

$$
0=\triangle \mu_{\mathrm{o}}=\triangle F^{\prime}\left(u_{\mathrm{o}}\right)
$$

since $\mu_{\mathrm{o}}=F^{\prime}\left(u_{\mathrm{o}}\right)$. The $O(\varepsilon)$ problem is

$$
\frac{\partial u_{\mathrm{o}}}{\partial \tau}=\Delta \mu_{1}=\Delta\left(F^{\prime \prime}\left(u_{\mathrm{o}}\right) u_{1}\right)
$$

and for the $O\left(\varepsilon^{2}\right)$ terms we obtain

$$
\frac{\partial u_{1}}{\partial \tau}=\Delta \mu_{2}=\Delta\left(F^{\prime \prime}\left(u_{\mathrm{o}}\right) u_{2}+\frac{1}{2} F^{\prime \prime \prime}\left(u_{\mathrm{o}}\right) u_{1}^{2}-2 A_{k l}\left(\tilde{u}_{\mathrm{o}}\right) \frac{\partial^{2} \tilde{u}_{\mathrm{o}}}{\partial x_{k} \partial x_{l}}-A_{k l}^{\prime}\left(\tilde{u}_{\mathrm{o}}\right) \frac{\partial \tilde{u}_{\mathrm{o}}}{\partial x_{k}} \frac{\partial \tilde{u}_{\mathrm{o}}}{\partial x_{l}}\right)
$$

plus corresponding boundary conditions on $\partial \Omega$. The boundary conditions on $\Gamma_{k}$ will be obtained via matching to the solution of the "inner" problem valid in the vicinity of the interface $\Gamma_{i}$.

\subsection{Inner problem}

3.2.1 Transformation to inner variables. We consider the 2D situation, where $\boldsymbol{x}=\left(x_{1}, x_{2}\right)=$ $(x, y)$. Let $\boldsymbol{r}(\tau, s)=\left(r_{1}(\tau, s), r_{2}(\tau, s)\right)$ be a parametrization of the curve $\Gamma_{k}$, where $s$ denotes arclength. Then

$$
\boldsymbol{x}(\tau, s, z)=\boldsymbol{r}(\tau, s)+\varepsilon z \boldsymbol{\nu}(\tau, s)
$$

defines the boundary layer with $z$ being the boundary layer or "inner" variable (see Fig. 4). The normal

$$
\boldsymbol{\nu}(\tau, s)=\left(-\frac{\partial r_{2}}{\partial s}(\tau, s), \frac{\partial r_{1}}{\partial s}(\tau, s)\right)
$$

points inside the precipitate and the tangent vector

$$
\boldsymbol{t}(\tau, s)=\left(\frac{\partial r_{1}}{\partial s}(\tau, s), \frac{\partial r_{2}}{\partial s}(\tau, s)\right)
$$

points in the counter-clockwise direction. 


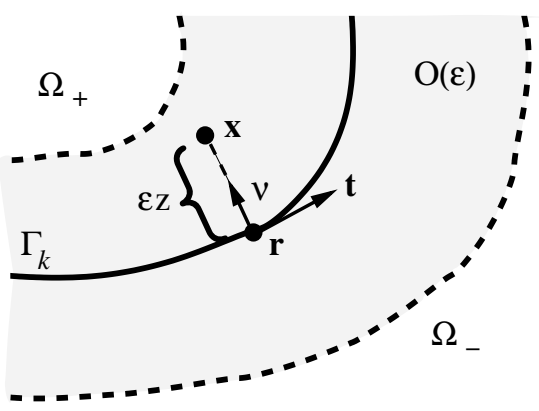

FIG. 4. Boundary-layer region.

Suppose $\tilde{w}$ is a quantity defined in the inner coordinates $(s, z, \tau)$. Then its derivatives are related to the derivatives of the corresponding quantity $w$ in outer coordinates via the invertible transformation matrix

$$
M=\left(\begin{array}{ccc}
Q & 0 \\
\frac{\partial x}{\partial \tau} & \frac{\partial y}{\partial \tau} & 1
\end{array}\right), \quad \text { where } \quad Q=\left(\begin{array}{ll}
\frac{\partial x}{\partial s} & \frac{\partial y}{\partial s} \\
\frac{\partial x}{\partial z} & \frac{\partial y}{\partial z}
\end{array}\right),
$$

by

Since $s$ is arclength,

$$
\left(\begin{array}{l}
\frac{\partial \tilde{w}}{\partial s} \\
\frac{\partial \tilde{w}}{\partial z} \\
\frac{\partial \tilde{w}}{\partial \tau}
\end{array}\right)=M\left(\begin{array}{l}
\frac{\partial w}{\partial x} \\
\frac{\partial w}{\partial y} \\
\frac{\partial w}{\partial \tau}
\end{array}\right)
$$

$$
\left(\frac{\partial r_{1}}{\partial s}\right)^{2}+\left(\frac{\partial r_{2}}{\partial s}\right)^{2}=1, \quad \frac{\partial r_{1}}{\partial s} \frac{\partial^{2} r_{1}}{\partial s^{2}}+\frac{\partial r_{2}}{\partial s} \frac{\partial^{2} r_{2}}{\partial s^{2}}=0
$$

and for the curvature we have

$$
\kappa(s, \tau)=\frac{\partial r_{1}}{\partial s} \frac{\partial^{2} r_{2}}{\partial s^{2}}+\frac{\partial r_{2}}{\partial s} \frac{\partial^{2} r_{1}}{\partial s^{2}}
$$

so that

$$
\frac{\partial \mathbf{t}}{\partial s}=\kappa \boldsymbol{\nu}, \quad \frac{\partial \boldsymbol{\nu}}{\partial s}=-\kappa \mathbf{t}
$$

and

$$
\frac{\partial^{2} r_{1}}{\partial s^{2}}=-\kappa \frac{\partial r_{2}}{\partial s}, \quad \frac{\partial^{2} r_{2}}{\partial s^{2}}=\kappa \frac{\partial r_{1}}{\partial s} .
$$

Hence,

$$
\frac{\partial \boldsymbol{x}}{\partial s}=(1-\varepsilon \kappa) \mathbf{t}, \quad \frac{\partial \boldsymbol{x}}{\partial z}=\varepsilon \boldsymbol{\nu}, \quad \operatorname{det} Q=\varepsilon(1-\varepsilon z \kappa) .
$$


Now we can express the quantity $w$ in the outer variables in terms of the inner variables by

$$
\left(\begin{array}{c}
\frac{\partial w}{\partial x} \\
\frac{\partial w}{\partial y} \\
\frac{\partial w}{\partial \tau}
\end{array}\right)=\left(\begin{array}{cc}
Q^{-1} & 0 \\
-\frac{\partial \boldsymbol{x}}{\partial \tau} \cdot Q^{-1} & 1
\end{array}\right)\left(\begin{array}{l}
\frac{\partial \tilde{w}}{\partial s} \\
\frac{\partial \tilde{w}}{\partial z} \\
\frac{\partial \tilde{w}}{\partial \tau}
\end{array}\right)=\left(\begin{array}{ccc}
(1+\varepsilon z \kappa) \frac{\partial r_{1}}{\partial s} & -\varepsilon^{-1} \frac{\partial r_{2}}{\partial s} & 0 \\
(1+\varepsilon z \kappa) \frac{\partial r_{2}}{\partial s} & \varepsilon^{-1} \frac{\partial r_{1}}{\partial s} & 0 \\
-(1+\varepsilon z \kappa) V^{\mathbf{t}} & -\varepsilon^{-1} V^{\nu} & 1
\end{array}\right)\left(\begin{array}{l}
\frac{\partial \tilde{w}}{\partial s} \\
\frac{\partial \tilde{w}}{\partial z} \\
\frac{\partial \tilde{w}}{\partial \tau}
\end{array}\right),
$$

where we have used the approximation $1 /(1-\varepsilon z \kappa)=1+\varepsilon z \kappa+O\left(\varepsilon^{2}\right)$, and where we denote the tangential and normal velocity by

$$
V^{\mathbf{t}}=\frac{\partial \boldsymbol{x}}{\partial \tau} \cdot \mathbf{t}, \quad V^{\boldsymbol{\nu}}=\frac{\partial \boldsymbol{x}}{\partial \tau} \cdot \boldsymbol{\nu},
$$

respectively.

The higher derivatives then transform as follows:

$$
\begin{aligned}
\frac{\partial^{2} w}{\partial x^{2}}= & \varepsilon^{-2}\left(\frac{\partial r_{2}}{\partial s}\right)^{2} \frac{\partial^{2} \tilde{w}}{\partial z^{2}}-\varepsilon^{-1}\left[\kappa\left(\frac{\partial r_{1}}{\partial s}\right)^{2} \frac{\partial \tilde{w}}{\partial z}+2 \frac{\partial r_{1}}{\partial s} \frac{\partial r_{2}}{\partial s} \frac{\partial^{2} \tilde{w}}{\partial s \partial z}\right]+\left(\frac{\partial r_{1}}{\partial s}\right)^{2} \frac{\partial^{2} \tilde{w}}{\partial s^{2}} \\
& -2 \kappa \frac{\partial r_{1}}{\partial s} \frac{\partial r_{2}}{\partial s} \frac{\partial \tilde{w}}{\partial s}-z \kappa\left[\kappa\left(\frac{\partial r_{1}}{\partial s}\right)^{2} \frac{\partial \tilde{w}}{\partial z}+2 \frac{\partial r_{1}}{\partial s} \frac{\partial r_{2}}{\partial s} \frac{\partial^{2} \tilde{w}}{\partial s \partial z}\right], \\
\frac{\partial^{2} w}{\partial y^{2}}= & \varepsilon^{-2}\left(\frac{\partial r_{1}}{\partial s}\right)^{2} \frac{\partial^{2} \tilde{w}}{\partial z^{2}}-\varepsilon^{-1}\left[\kappa\left(\frac{\partial r_{2}}{\partial s}\right)^{2} \frac{\partial \tilde{w}}{\partial z}-2 \frac{\partial r_{1}}{\partial s} \frac{\partial r_{2}}{\partial s} \frac{\partial^{2} \tilde{w}}{\partial s \partial z}\right]+\left(\frac{\partial r_{2}}{\partial s}\right)^{2} \frac{\partial^{2} \tilde{w}}{\partial s^{2}} \\
& +2 \kappa \frac{\partial r_{1}}{\partial s} \frac{\partial r_{2}}{\partial s} \frac{\partial \tilde{w}}{\partial s}-z \kappa\left[\kappa\left(\frac{\partial r_{2}}{\partial s}\right)^{2} \frac{\partial \tilde{w}}{\partial z}-2 \frac{\partial r_{1}}{\partial s} \frac{\partial r_{2}}{\partial s} \frac{\partial^{2} \tilde{w}}{\partial s \partial z}\right], \\
\frac{\partial^{2} w}{\partial x \partial y} & -\varepsilon^{-2} \frac{\partial r_{1}}{\partial s} \frac{\partial r_{2}}{\partial s} \frac{\partial^{2} \tilde{w}}{\partial z^{2}}-\varepsilon^{-1}\left[\kappa \frac{\partial r_{1}}{\partial s} \frac{\partial r_{2}}{\partial s} \frac{\partial \tilde{w}}{\partial z}+\left(\left(\frac{\partial r_{2}}{\partial s}\right)^{2}-\left(\frac{\partial r_{1}}{\partial s}\right)^{2}\right) \frac{\partial^{2} \tilde{w}}{\partial s \partial z}\right] \\
& +\frac{\partial r_{1}}{\partial s} \frac{\partial r_{2}}{\partial s} \frac{\partial^{2} \tilde{w}}{\partial s^{2}}-\kappa\left(\left(\frac{\partial r_{2}}{\partial s}\right)^{2}-\left(\frac{\partial r_{1}}{\partial s}\right)^{2}\right) \frac{\partial \tilde{w}}{\partial s} \\
& -z \kappa\left[\kappa \frac{\partial r_{1}}{\partial s} \frac{\partial r_{2}}{\partial s} \frac{\partial \tilde{w}}{\partial z}+\left(\left(\frac{\partial r_{2}}{\partial s}\right)^{2}-\left(\frac{\partial r_{1}}{\partial s}\right)^{2}\right) \frac{\partial^{2} \tilde{w}}{\partial s \partial z}\right], \\
\triangle w= & \varepsilon^{-2} \frac{\partial^{2} \tilde{w}}{\partial z^{2}}-\varepsilon^{-1} \kappa \frac{\partial \tilde{w}}{\partial z}+\frac{\partial^{2} \tilde{w}}{\partial s^{2}}-z \kappa^{2} \frac{\partial \tilde{w}}{\partial z} .
\end{aligned}
$$

3.2.2 Inner equations and matching. In what follows we write

$$
\tilde{u}_{\xi_{k}}:=\frac{\partial \tilde{u}}{\partial \xi_{k}}, \quad \tilde{u}_{\xi_{k} \xi_{l}}:=\frac{\partial^{2} \tilde{u}}{\partial \xi_{k} \partial \xi_{l}}, \quad \tilde{u}_{\mathrm{o} \xi_{k}}:=\frac{\partial \tilde{u}_{\mathrm{o}}}{\partial \xi_{k}}, \quad \tilde{u}_{\mathrm{o} \xi_{k} \xi_{l}}:=\frac{\partial^{2} \tilde{u}_{\mathrm{o}}}{\partial \xi_{k} \partial \xi_{l}} .
$$

Making use of 3.19-3.29 we can expand the gradient energy part in inner coordinates:

$$
2 A_{k l}(u) \frac{\partial^{2} u}{\partial x_{k} \partial x_{l}}+A_{k l}^{\prime}(u) \frac{\partial u}{\partial x_{k}} \frac{\partial u}{\partial x_{l}}=\varepsilon^{-2} g\left(\tilde{u}, \tilde{u}_{\xi_{k}}, \tilde{u}_{\xi_{k} \xi_{l}}\right)+\varepsilon^{-1} h\left(\tilde{u}, \tilde{u} \xi_{k}, \tilde{u} \xi_{k} \xi_{l}\right)+j\left(\tilde{u}, \tilde{u}_{\xi_{k}}, \tilde{u}_{\xi_{k} \xi_{l}}\right),
$$


where $\xi_{k}, \xi_{l} \in\{s, z\}$ and

$$
\begin{aligned}
& g\left(\tilde{u}, \tilde{u}_{\xi_{k}}, \tilde{u}_{\xi_{k} \xi_{l}}\right)=2 \boldsymbol{\nu} A \boldsymbol{\nu}^{\dagger} \frac{\partial^{2} \tilde{u}}{\partial z^{2}}+\boldsymbol{\nu} A^{\prime} \boldsymbol{\nu}^{\dagger}\left(\frac{\partial \tilde{u}}{\partial z}\right)^{2}, \\
& h\left(\tilde{u}, \tilde{u}_{\xi_{k}}, \tilde{u}_{\xi_{k} \xi_{l}}\right)=-2 \kappa \mathbf{t} A \mathbf{t}^{\dagger} \frac{\partial \tilde{u}}{\partial z}+2\left(\mathbf{t} A \boldsymbol{\nu}^{\dagger}+\boldsymbol{\nu} A \mathbf{t}^{\dagger}\right) \frac{\partial^{2} \tilde{u}}{\partial s \partial z}+\left(\mathbf{t} A^{\prime} \boldsymbol{\nu}^{\dagger}+\boldsymbol{\nu} A^{\prime} \mathbf{t}^{\dagger}\right) \frac{\partial \tilde{u}}{\partial s} \frac{\partial \tilde{u}}{\partial z}, \\
& j\left(\tilde{u}, \tilde{u}_{\xi_{k}}, \tilde{u}_{\xi_{k} \xi_{l}}\right)=2 \mathbf{t} A \mathbf{t}^{\dagger} \frac{\partial^{2} \tilde{u}}{\partial s^{2}}+2 \kappa\left(\mathbf{t} A^{\prime} \boldsymbol{\nu}^{\dagger}+\boldsymbol{\nu} A^{\prime} \mathbf{t}^{\dagger}\right) \frac{\partial \tilde{u}}{\partial s}+\mathbf{t} A \mathbf{t}^{\dagger} \frac{\partial \tilde{u}^{2}}{\partial s}+z \kappa h\left(\tilde{u}, \tilde{u} \xi_{k}, \tilde{u} \xi_{k} \xi_{l}\right)
\end{aligned}
$$

with

$$
A(\tilde{u})=\left(\begin{array}{ll}
A_{11}(\tilde{u}) & A_{12}(\tilde{u}) \\
A_{21}(\tilde{u}) & A_{22}(\tilde{u})
\end{array}\right),
$$

and the superscript $\dagger$ denotes the transpose of a vector.

From this we obtain

$$
\begin{aligned}
\triangle \mu= & \varepsilon^{-2} \frac{\partial^{2}}{\partial z^{2}}\left[F^{\prime}(\tilde{u})-g\left(\tilde{u}, \tilde{u}_{\xi_{k}}, \tilde{u}_{\xi_{k} \xi_{l}}\right)\right] \\
& -\varepsilon^{-1}\left(\frac{\partial^{2} h}{\partial z^{2}}\left(\tilde{u}, \tilde{u}_{\xi_{k}}, \tilde{u}_{\xi_{k} \xi_{l}}\right)-\kappa \frac{\partial}{\partial z}\left[F^{\prime}(\tilde{u})-g\left(\tilde{u}, \tilde{u}_{\xi_{k}}, \tilde{u}_{\xi_{k} \xi_{l}}\right)\right]\right) \\
& -\frac{\partial^{2} j}{\partial z^{2}}\left(\tilde{u}, \tilde{u} \xi_{k}, \tilde{u}_{\xi_{k} \xi_{l}}\right)-\kappa \frac{\partial h}{\partial z}\left(\tilde{u}, \tilde{u}_{\xi_{k}}, \tilde{u}_{\xi_{k} \xi_{l}}\right)+\frac{\partial^{2}}{\partial s^{2}}\left[F^{\prime}(\tilde{u})-g\left(\tilde{u}, \tilde{u}_{\xi_{k}}, \tilde{u}_{\xi_{k} \xi_{l}}\right)\right] \\
& -z \kappa^{2} \frac{\partial}{\partial z}\left[F^{\prime}(\tilde{u})-g\left(\tilde{u}, \tilde{u}_{\xi_{k}}, \tilde{u}_{\xi_{k} \xi_{l}}\right)\right]+O(\varepsilon) .
\end{aligned}
$$

Let the quantities $u$ and $\mu$ have inner expansions

$$
\begin{aligned}
& \tilde{u}(\tau, s, z)=\tilde{u}_{\mathrm{o}}(\tau, s, z)+\varepsilon \tilde{u}_{1}(\tau, s, z)+\varepsilon^{2} \tilde{u}_{2}(\tau, s, z)+O\left(\varepsilon^{3}\right), \\
& \tilde{\mu}(\tau, s, z)=\tilde{\mu}_{\mathrm{o}}(\tau, s, z)+\varepsilon \tilde{\mu}_{1}(\tau, s, z)+\varepsilon^{2} \tilde{\mu}_{2}(\tau, s, z)+O\left(\varepsilon^{3}\right) .
\end{aligned}
$$

The function $F(\tilde{u})$ can be expanded as

$$
F^{\prime}(\tilde{u})=F^{\prime}\left(\tilde{u}_{\mathrm{o}}\right)+\varepsilon \tilde{u}_{1} F^{\prime \prime}\left(\tilde{u}_{\mathrm{o}}\right)+\varepsilon^{2}\left(\frac{1}{2} \tilde{u}_{1}^{2} F^{\prime \prime \prime}\left(\tilde{u}_{\mathrm{o}}\right)+\tilde{u}_{2} F^{\prime \prime}\left(\tilde{u}_{\mathrm{o}}\right)\right),
$$

and $A(\tilde{u})$ can be expanded similarly.

Then, using 3.29 and 3.36 the leading order inner $\left(O\left(\varepsilon^{-2}\right)\right)$ problem is

$$
\frac{\partial^{2} \tilde{u}_{\mathrm{o}}}{\partial z^{2}}=\frac{\partial^{2}}{\partial z^{2}}\left[F^{\prime}\left(\tilde{u}_{\mathrm{o}}\right)-g\left(\tilde{u}_{\mathrm{o}}, \tilde{u}_{\mathrm{o} \xi_{k}}, \tilde{u}_{\mathrm{o} \xi_{k} \xi_{l}}\right)\right]=0,
$$

so that

$$
\tilde{\mu}_{\mathrm{o}}(\tau, \boldsymbol{r}, z)=a_{\mathrm{o}}(\tau, \boldsymbol{r}) z+b_{\mathrm{o}}(\tau, \boldsymbol{r}) .
$$

Recall that the leading order outer problem for $\mu$ is

$$
\begin{aligned}
\Delta \mu_{\mathrm{o}}^{-}=0 & \text { in } \Omega_{-}, \\
\boldsymbol{n} \cdot \nabla \mu_{\mathrm{o}}^{-}=0 & \text { on } \partial \Omega_{-}, \\
\Delta \mu_{\mathrm{o}}^{+}=0 & \text { in } \Omega_{+},
\end{aligned}
$$


where $\mu_{\mathrm{o}}^{-}$and $\mu_{\mathrm{o}}^{+}$denote the chemical potential in the matrix and the precipitate, respectively. Both problems have to be joined by a condition on the interface $\Gamma_{k}$ of the precipitate. This will be provided by matching with the inner solution. For this we express the outer solution in inner coordinates and reexpand

$$
\begin{aligned}
\left(\mu_{\mathrm{o}}^{ \pm}+\varepsilon \mu_{1}^{ \pm}+\right. & \left.\varepsilon \mu_{2}^{ \pm}+O\left(\varepsilon^{3}\right)\right)(\tau, \boldsymbol{r}+\varepsilon z \boldsymbol{\nu})=\mu_{\mathrm{o}}^{ \pm}(\tau, \boldsymbol{r})+\varepsilon\left(\mu_{1}^{ \pm}(\tau, \boldsymbol{r})+z \boldsymbol{\nu} \cdot \boldsymbol{\nabla}_{\boldsymbol{x}} \mu_{\mathrm{o}}^{ \pm}(\tau, \boldsymbol{r})\right) \\
& +\varepsilon^{2}\left(\mu_{2}^{ \pm}(\tau, \boldsymbol{r})+z \boldsymbol{\nu} \cdot \nabla_{\boldsymbol{x}} \mu_{1}^{ \pm}(\tau, \boldsymbol{r})+\frac{z^{2}}{2} \boldsymbol{\nu} H\left(\mu_{\mathrm{o}}^{ \pm}(\tau, \boldsymbol{r})\right) \boldsymbol{\nu}^{T}\right)+O\left(\varepsilon^{3}\right),
\end{aligned}
$$

where

$$
H(w):=\left(\begin{array}{ll}
\frac{\partial^{2} w}{\partial x^{2}} & \frac{\partial^{2} w}{\partial x \partial y} \\
\frac{\partial^{2} w}{\partial y \partial x} & \frac{\partial^{2} w}{\partial y^{2}}
\end{array}\right)
$$

Matching to leading order then requires

$$
\mu_{\mathrm{o}}^{ \pm}(\tau, \boldsymbol{r})=\lim _{z \rightarrow \pm \infty} \tilde{\mu}_{\mathrm{o}}(\tau, \boldsymbol{r}, z)
$$

as $\varepsilon \rightarrow 0$. Therefore $a_{\mathrm{o}}(\tau, \boldsymbol{r})=0$ and $\mu_{\mathrm{o}}^{ \pm}(\tau, \boldsymbol{r})=b_{\mathrm{o}}(\tau, \boldsymbol{r})$.

Furthermore, from the ordinary differential equation for $u_{\mathrm{o}}$ in $z, 3.40$ and 3.32, we obtain

$$
\left(2 \boldsymbol{\nu} A\left(\tilde{u}_{\mathrm{o}}\right) \boldsymbol{\nu}^{\dagger}\right) \frac{\partial^{2} \tilde{u}_{\mathrm{o}}}{\partial z^{2}}+\left(\boldsymbol{\nu} A^{\prime}\left(\tilde{u}_{\mathrm{o}}\right) \boldsymbol{\nu}^{\dagger}\right)\left(\frac{\partial \tilde{u}_{\mathrm{o}}}{\partial z}\right)^{2}=F^{\prime}\left(\tilde{u}_{\mathrm{o}}\right)-b_{\mathrm{o}}(\tau, \boldsymbol{r})
$$

Hence we find

$$
\begin{aligned}
\frac{1}{2} \frac{\mathrm{d}}{\mathrm{d} z}\left[\left(2 \boldsymbol{\nu} A\left(\tilde{u}_{\mathrm{o}}\right) \boldsymbol{\nu}^{\dagger}\right)\left(\frac{\partial \tilde{u}_{\mathrm{o}}}{\partial z}\right)^{2}\right] & =\left[\left(2 \boldsymbol{\nu} A\left(\tilde{u}_{\mathrm{o}}\right) \boldsymbol{\nu}^{\dagger}\right) \frac{\partial^{2} \tilde{u}_{\mathrm{o}}}{\partial z^{2}}+\left(\boldsymbol{\nu} A^{\prime}\left(\tilde{u}_{\mathrm{o}}\right) \boldsymbol{\nu}^{\dagger}\right) \frac{\partial \tilde{u}_{\mathrm{o}}}{\partial z}\right]\left(\frac{\partial \tilde{u}_{\mathrm{o}}}{\partial z}\right)^{2} \\
& =\left(F^{\prime}\left(\tilde{u}_{\mathrm{o}}\right)-b_{\mathrm{o}}(\tau, \boldsymbol{r})\right) \frac{\partial \tilde{u}_{\mathrm{o}}}{\partial z} .
\end{aligned}
$$

This yields, after integrating from $z=+\infty$ to $z=-\infty$ and observing that $\partial \tilde{u}_{\mathrm{o}} / \partial z \rightarrow 0$ as $z \rightarrow \pm \infty$, the solvability condition

$$
\int_{u_{-}}^{u_{+}}\left[F^{\prime}\left(\tilde{u}_{\mathrm{o}}\right)-b_{\mathrm{o}}(\tau, \boldsymbol{r})\right] \mathrm{d} \tilde{u}_{\mathrm{o}}=0 \quad \text { with } \quad F^{\prime}\left(u_{ \pm}\right)=b_{\mathrm{o}}
$$

Equation (3.48) is basically a system of three equations in three unknowns $u_{+}, u_{-}$and $b_{0}$. It means that for a given free energy $F(u)$ of the form of a double well, $b_{\mathrm{o}}$ is the unique constant such that the integral in 3.48, i.e. the "area" in Figure 2 between $u_{-}$and $u_{+}$, is zero.

Finally, the uniqueness of solution to the problem

$$
\begin{aligned}
\Delta \mu_{\mathrm{o}}^{-}=0 & \text { in } \Omega_{-}, \\
\boldsymbol{n} \cdot \nabla \mu_{\mathrm{o}}^{-}=0 & \text { on } \partial \Omega_{-}, \\
\mu_{\mathrm{o}}^{ \pm}=b_{\mathrm{o}} & \text { on } \Gamma_{k}, \\
\Delta \mu_{\mathrm{o}}^{+}=0 & \text { in } \Omega_{+},
\end{aligned}
$$

ensures that $F^{\prime}\left(u_{\mathrm{o}}\right)=\mu_{\mathrm{o}}=$ const in all of $\Omega$. 
To order $O\left(\varepsilon^{-1}\right)$ we obtain for the inner problem

$$
\begin{aligned}
0= & -\frac{\partial^{2} h}{\partial z^{2}}\left(\tilde{u}_{\mathrm{o}}, \tilde{u}_{\mathrm{o} \xi_{k}}, \tilde{u}_{\mathrm{o} \xi_{k} \xi_{l}}\right)+\kappa \frac{\partial \tilde{\mu}_{\mathrm{o}}}{\partial z}+\frac{\partial^{2}}{\partial z^{2}}\left[F^{\prime \prime}\left(\tilde{u}_{\mathrm{o}}\right) \tilde{u}_{1}-2 \boldsymbol{\nu} A\left(\tilde{u}_{\mathrm{o}}\right) \boldsymbol{\nu}^{\dagger} \frac{\partial^{2} \tilde{u}_{1}}{\partial z^{2}}\right. \\
& \left.-2 \boldsymbol{\nu} A^{\prime}\left(\tilde{u}_{\mathrm{o}}\right) \tilde{u}_{1} \boldsymbol{\nu}^{\dagger} \frac{\partial^{2} \tilde{u}_{\mathrm{o}}}{\partial z^{2}}-2 \boldsymbol{\nu} A^{\prime} \boldsymbol{\nu}^{\dagger} \frac{\partial \tilde{u}_{\mathrm{o}}}{\partial z} \frac{\partial \tilde{u}_{1}}{\partial z}+\boldsymbol{\nu} A^{\prime \prime}\left(\tilde{u}_{\mathrm{o}}\right) \tilde{u}_{1} \boldsymbol{\nu}^{\dagger}\left(\frac{\partial \tilde{u}_{\mathrm{o}}}{\partial z}\right)^{2}\right] \\
= & -\frac{\partial^{2} h}{\partial z^{2}}\left(\tilde{u}_{\mathrm{o}}, \tilde{u}_{\mathrm{o} \xi_{k}}, \tilde{u}_{\mathrm{o} \xi_{k} \xi_{l}}\right)+\kappa \frac{\partial \tilde{\mu}_{\mathrm{o}}}{\partial z}+\frac{\partial^{2}}{\partial z^{2}}\left[\lim _{\varepsilon \rightarrow 0} \frac{\mathrm{d}}{\mathrm{d} \varepsilon}\left(F^{\prime}(\tilde{u})-g\left(\tilde{u}, \tilde{u}_{\xi_{k}}, \tilde{u}_{\xi_{k} \xi_{l}}\right)\right)\right] \\
= & \kappa \frac{\partial \tilde{\mu}_{\mathrm{o}}}{\partial z}+\frac{\partial^{2} \tilde{\mu}_{1}}{\partial z^{2}} .
\end{aligned}
$$

Note $\tilde{\mu}_{\mathrm{o}}$ is constant so that $\kappa \partial \tilde{\mu}_{\mathrm{o}} / \partial z=0$ and the right hand side of 3.50 is equal to $\partial^{2} \tilde{\mu}_{1} / \partial z^{2}$. Hence,

$$
\tilde{\mu}_{1}(\tau, \boldsymbol{r}, z)=a_{1}(\tau, \boldsymbol{r}) z+b_{1}(\tau, \boldsymbol{r}) .
$$

Moreover, since $\mu_{\mathrm{o}}$ is constant, matching to next order yields

$$
\mu_{1}^{ \pm}(\tau, \boldsymbol{r})=\lim _{z \rightarrow \pm \infty} \tilde{\mu}_{1}(\tau, \boldsymbol{r}, z)
$$

Hence, $a_{1}(\tau, \boldsymbol{r})=0$ and $\tilde{\mu}_{1}=b_{1}(\tau, \boldsymbol{r})$ are independent of $z$ and

$$
\tilde{\mu}_{1}(\tau, \boldsymbol{r})=-h\left(\tilde{u}_{\mathrm{o}}, \tilde{u}_{\mathrm{o} \xi_{k}}, \tilde{u}_{\mathrm{o} \xi_{k} \xi_{l}}\right)+\lim _{\varepsilon \rightarrow 0} \frac{\mathrm{d}}{\mathrm{d} \varepsilon}\left(F^{\prime}(\tilde{u})-g\left(\tilde{u}, \tilde{u}_{\xi_{k}}, \tilde{u}_{\xi_{k} \xi_{l}}\right)\right) .
$$

Note now that

$$
\begin{aligned}
\frac{\partial}{\partial s}\left[\left(\mathbf{t} A \boldsymbol{\nu}^{\dagger}+\boldsymbol{\nu} A \mathbf{t}^{\dagger}\right)\left(\frac{\partial \tilde{u}_{\mathrm{o}}}{\partial z}\right)^{2}\right]= & \left(\mathbf{t} A \boldsymbol{\nu}^{\dagger}+\boldsymbol{\nu} A \mathbf{t}^{\dagger}\right) 2 \frac{\partial \tilde{u}_{\mathrm{o}}}{\partial z} \frac{\partial^{2} \tilde{u}_{\mathrm{o}}}{\partial s \partial z}+\left(\mathbf{t} A^{\prime} \boldsymbol{\nu}^{\dagger}+\boldsymbol{\nu} A^{\prime} \boldsymbol{\nu}^{\dagger}\right) \frac{\partial \tilde{u}_{\mathrm{o}}}{\partial s}\left(\frac{\partial \tilde{u}_{\mathrm{o}}}{\partial z}\right)^{2} \\
& -2 \kappa\left(\mathbf{t} A \mathbf{t}^{\dagger}-\boldsymbol{\nu} A \boldsymbol{\nu}^{\dagger}\right)\left(\frac{\partial \tilde{u}_{\mathrm{o}}}{\partial z}\right)^{2}
\end{aligned}
$$

This implies

$$
\begin{aligned}
\frac{\partial \tilde{u}_{\mathrm{o}}}{\partial z} \tilde{\mu}_{1}(\tau, \boldsymbol{r})= & 2 \kappa \boldsymbol{\nu} A \boldsymbol{\nu}^{\dagger}\left(\frac{\partial \tilde{u}_{\mathrm{o}}}{\partial z}\right)^{2}-\frac{\partial}{\partial s}\left[\left(\mathbf{t} A \boldsymbol{\nu}^{\dagger}+\boldsymbol{\nu} A \mathbf{t}^{\dagger}\right)\left(\frac{\partial \tilde{u}_{\mathrm{o}}}{\partial z}\right)^{2}\right] \\
& +\frac{\partial \tilde{u}_{\mathrm{o}}}{\partial z} \lim _{\varepsilon \rightarrow 0} \frac{\mathrm{d}}{\mathrm{d} \varepsilon}\left(F^{\prime}(\tilde{u})-g\left(\tilde{u}, \tilde{u}_{\xi_{k}}, \tilde{u}_{\xi_{k} \xi_{l}}\right)\right) .
\end{aligned}
$$

The condition joining the two outer problems for $\mu_{1}$ is now obtained by integrating (3.55) from $z=-\infty$ to $z=+\infty$. First we observe that the third term on the right hand side vanishes. For this recall that

$$
\begin{aligned}
\frac{\partial \tilde{u}_{\mathrm{o}}}{\partial z} \lim _{\varepsilon \rightarrow 0} \frac{\mathrm{d}}{\mathrm{d} \varepsilon}\left(F^{\prime}(\tilde{u})-g\left(\tilde{u}, \tilde{u}_{\xi_{k}}, \tilde{u}_{\xi_{k} \xi_{l}}\right)\right)= & F^{\prime \prime}\left(\tilde{u}_{\mathrm{o}}\right) \frac{\partial \tilde{u}_{\mathrm{o}}}{\partial z} \tilde{u}_{1}-2 \boldsymbol{\nu} A \boldsymbol{\nu}^{\dagger} \frac{\partial^{2} \tilde{u}_{1}}{\partial z^{2}} \frac{\partial \tilde{u}_{\mathrm{o}}}{\partial z}-\boldsymbol{\nu} A^{\prime \prime} \boldsymbol{\nu}^{\dagger} \tilde{u}_{1}\left(\frac{\partial \tilde{u}_{\mathrm{o}}}{\partial z}\right)^{3} \\
& -2 \boldsymbol{\nu} A^{\prime} \boldsymbol{\nu}^{\dagger}\left(\frac{\partial \tilde{u}_{\mathrm{o}}}{\partial z}\right)^{2} \frac{\partial \tilde{u}_{1}}{\partial z}-2 \boldsymbol{\nu} A^{\prime} \boldsymbol{\nu}^{\dagger} \tilde{u}_{1} \frac{\partial \tilde{u}_{\mathrm{o}}}{\partial z} \frac{\partial^{2} \tilde{u}_{\mathrm{o}}}{\partial z^{2}}
\end{aligned}
$$


and note that 3.46 implies

$$
\begin{aligned}
F^{\prime \prime}\left(\tilde{u}_{\mathrm{o}}\right) \frac{\partial \tilde{u}_{\mathrm{o}}}{\partial z} \tilde{u}_{1}= & 2 \boldsymbol{\nu} A^{\prime} \boldsymbol{\nu}^{\dagger} \tilde{u}_{1} \frac{\partial \tilde{u}_{\mathrm{o}}}{\partial z} \frac{\partial^{2} \tilde{u}_{\mathrm{o}}}{\partial z^{2}}+2 \boldsymbol{\nu} A \boldsymbol{\nu}^{\dagger} \tilde{u}_{1} \frac{\partial^{3} \tilde{u}_{\mathrm{o}}}{\partial z^{3}} \\
& +\boldsymbol{\nu} A^{\prime \prime} \boldsymbol{\nu}^{\dagger} \tilde{u}_{1}\left(\frac{\partial \tilde{u}_{\mathrm{o}}}{\partial z}\right)^{3}+2 \boldsymbol{\nu} A^{\prime} \boldsymbol{\nu}^{\dagger} \tilde{u}_{1} \frac{\partial \tilde{u}_{\mathrm{o}}}{\partial z} \frac{\partial^{2} \tilde{u}_{\mathrm{o}}}{\partial z^{2}}
\end{aligned}
$$

Hence

$$
\frac{\partial \tilde{u}_{\mathrm{o}}}{\partial z} \lim _{\varepsilon \rightarrow 0} \frac{\mathrm{d}}{\mathrm{d} \varepsilon}\left(F^{\prime}(\tilde{u})-g\left(\tilde{u}, \tilde{u}_{\xi_{k}}, \tilde{u}_{\xi_{k} \xi_{l}}\right)\right)=\frac{\partial}{\partial z}\left[2 \boldsymbol{\nu} A \boldsymbol{\nu}^{\dagger}\left(\tilde{u}_{1} \frac{\partial^{2} \tilde{u}_{\mathrm{o}}}{\partial z^{2}}-\frac{\partial \tilde{u}_{1}}{\partial z} \frac{\partial \tilde{u}_{\mathrm{o}}}{\partial z}\right)\right],
$$

the integral of which from $z=-\infty$ to $z=+\infty$ vanishes since $\partial \tilde{u}_{\mathrm{o}} / \partial z, \partial^{2} \tilde{u}_{\mathrm{o}} / \partial z^{2} \rightarrow 0$ as $z \rightarrow$ $\pm \infty$. For the integral of the other two terms on the right hand side of (3.55), we define

$$
G\left(\tilde{u}_{\mathrm{o}}\right)=\int_{u_{-}}^{\tilde{u}_{\mathrm{o}}}\left[F^{\prime}(v)-b_{\mathrm{o}}\right] \mathrm{d} v
$$

and observe that from 3.46 ,

$$
\frac{\partial \tilde{u}_{\mathrm{o}}}{\partial z}=\sqrt{\frac{G\left(\tilde{u}_{\mathrm{o}}\right)}{\nu A \nu^{\dagger}}}
$$

so that the integral of 3.55 can be written

$$
\left[\left[u_{\mathrm{o}}^{ \pm}\right]\right] \tilde{\mu}_{1}(\tau, \boldsymbol{r})=2 \kappa \int_{u_{-}}^{u_{+}} \sqrt{\boldsymbol{\nu} A \boldsymbol{\nu}^{\dagger} G(v)} \mathrm{d} v-\frac{\partial}{\partial s} \int_{u_{-}}^{u_{+}}\left(\mathbf{t} A \boldsymbol{\nu}^{\dagger}+\boldsymbol{\nu} A \mathbf{t}^{\dagger}\right) \sqrt{\frac{G(v)}{\nu A \boldsymbol{\nu}^{\dagger}}} \mathrm{d} v
$$

where $\left[\left[u_{\mathrm{o}}^{ \pm}\right]\right]$denotes the jump $u_{\mathrm{o}}^{+}-u_{\mathrm{o}}^{-}$. This can also be written, after differentiation with respect to $s$, as

$$
\tilde{\mu}_{1}(\tau, \boldsymbol{r})=\frac{\kappa}{\left[\left[u_{\mathrm{o}}^{ \pm}\right]\right]}\left(2 \int_{u_{-}}^{u_{+}} \mathbf{t} A \mathbf{t}^{\dagger} \sqrt{\frac{G(v)}{\boldsymbol{\nu} A \boldsymbol{\nu}^{\dagger}}} \mathrm{d} v-\frac{1}{2} \int_{u_{-}}^{u_{+}}\left(\mathbf{t} A \boldsymbol{\nu}^{\dagger}+\boldsymbol{\nu} A \mathbf{t}^{\dagger}\right)^{2} \sqrt{\frac{G(v)}{\left(\boldsymbol{\nu} A \boldsymbol{\nu}^{\dagger}\right)^{3}}} \mathrm{~d} v\right)
$$

By the matching condition (3.52) this equals $\mu_{1}(\tau, \boldsymbol{r})$. Given $F(u)$ and $A(u)$ it represents the chemical potential along the interface $\Gamma_{k}$ of a precipitate. This leads to the sharp interface model

$$
\begin{aligned}
\triangle \mu_{1}^{-} & =0 \quad \text { in } \Omega_{-}, \\
\boldsymbol{n} \cdot \nabla \mu_{1}^{-} & =0 \quad \text { on } \partial \Omega_{-}, \\
\mu_{1}^{ \pm} & =\tilde{\mu}_{1}(\tau, \boldsymbol{r}), \\
\triangle \mu_{1}^{+} & =0 \quad \text { in } \Omega_{+} .
\end{aligned}
$$

In order to determine the velocity of the sharp interface we have to continue the matching to higher order. To order $O(1)$ the inner problem reads 


$$
\begin{aligned}
-V^{\nu} \frac{\partial \tilde{u}_{\mathrm{o}}}{\partial z}= & -\frac{\partial^{2} j}{\partial z^{2}}\left(\tilde{u}_{\mathrm{o}}, \tilde{u}_{\mathrm{o} \xi_{k}}, \tilde{u}_{\mathrm{o} \xi_{k} \xi_{l}}\right)-\kappa \frac{\partial h}{\partial z}\left(\tilde{u}_{\mathrm{o}}, \tilde{u}_{\mathrm{o} \xi_{k}}, \tilde{u}_{\mathrm{o} \xi_{k} \xi_{l}}\right)+\frac{\partial^{2} \tilde{\mu}_{\mathrm{o}}}{\partial s^{2}}-z \kappa \frac{\partial \tilde{\mu}_{\mathrm{o}}}{\partial z} \\
& -\lim _{\varepsilon \rightarrow 0} \frac{\mathrm{d}}{\mathrm{d} \varepsilon}\left(h_{z z}\left(\tilde{u}, \tilde{u}_{\xi_{k}}, \tilde{u}_{\xi_{k} \xi_{l}}\right)-\kappa \frac{\partial}{\partial z}\left[F^{\prime}(\tilde{u})-g(\tilde{u})\right]\right) \\
& +\lim _{\varepsilon \rightarrow 0} \frac{\mathrm{d}^{2}}{\mathrm{~d} \varepsilon^{2}}\left(\frac{\partial^{2}}{\partial z^{2}}\left[F^{\prime}(\tilde{u})-g(\tilde{u})\right]\right), \\
= & \frac{\partial^{2} \tilde{\mu}_{2}}{\partial z^{2}}-\kappa \frac{\partial \tilde{\mu}_{1}}{\partial z}+\frac{\partial^{2} \tilde{\mu}_{\mathrm{o}}}{\partial s^{2}}-z \kappa \frac{\partial \tilde{\mu}_{\mathrm{o}}}{\partial z},
\end{aligned}
$$

where $V^{\boldsymbol{\nu}}=(\partial \boldsymbol{x} / \partial \tau) \cdot \boldsymbol{\nu}$. Again, since $\mu_{\mathrm{o}}$ is constant, we have

$$
\begin{aligned}
-V^{\nu} \frac{\partial \tilde{u}_{\mathrm{o}}}{\partial z} & =\frac{\partial^{2} \tilde{\mu}_{2}}{\partial z^{2}}-\kappa \frac{\partial}{\partial z}\left[h\left(\tilde{u}_{\mathrm{o}}, \tilde{u}_{\mathrm{o} \xi_{k}}, \tilde{u}_{\mathrm{o} \xi_{k} \xi_{l}}\right)-\lim _{\varepsilon \rightarrow 0} \frac{\mathrm{d}}{\mathrm{d} \varepsilon}\left(F^{\prime}(\tilde{u})-g\left(\tilde{u}, \tilde{u}_{\xi_{k}}, \tilde{u}_{\xi_{k} \xi_{l}}\right)\right)\right] \\
& =\frac{\partial^{2} \tilde{\mu}_{2}}{\partial z^{2}}-\kappa \frac{\partial \tilde{\mu}_{1}}{\partial z} .
\end{aligned}
$$

Since $\tilde{\mu}_{1}$ is independent of $z$, we simply have

$$
-V^{\nu} \frac{\partial \tilde{u}_{\mathrm{o}}}{\partial z}=\frac{\partial^{2} \tilde{\mu}_{2}}{\partial z^{2}} .
$$

We integrate 3.69 once with respect to $z$ and use the matching condition for $\partial \tilde{\mu}_{2} / \partial z$ to obtain

$$
\lim _{z \rightarrow \pm \infty} \frac{\partial \tilde{\mu}_{2}}{\partial z}=\boldsymbol{\nu} \cdot \nabla_{x} \mu_{1}^{ \pm}(\tau, \boldsymbol{r})
$$

The $z^{2}$-term in 3.43 vanishes since $\mu_{\mathrm{o}}$ is constant. Hence, for the interfacial speed we obtain

$$
V^{\nu}=-\frac{\left[\left[\boldsymbol{\nu} \cdot \nabla_{x} \mu_{1}^{ \pm}(\tau, \boldsymbol{r})\right]\right]}{\left[\left[u_{0}^{ \pm}\right]\right]} .
$$

Equations 3.63 - 3.66 together with 3.71 constitute our sharp-interface model, governing the long-time dynamics of the phase field model (3.4), 3.5) together with (3.7).

The sharp-interface model we obtained is very similar in structure to the one given in [25] and reduces to it when the surface energy is independent of concentration.

\subsection{The Cahn-Hoffmann law for concentration dependent surface energy}

We also obtain a Cahn-Hoffmann law for a concentration dependent surface energy [14], [3], [13]. To this end we define

$$
\boldsymbol{r}_{s}=(\cos \theta(\tau, s), \sin \theta(\tau, s)),
$$

where $\theta(\tau, s)$ is the angle of the tangent at a point on $\Gamma_{k}$ to the $x$-axis. In terms of this coordinate we have

$$
\frac{\partial^{2}}{\partial \theta^{2}}\left(\boldsymbol{\nu} A \boldsymbol{\nu}^{\dagger}\right)=2\left(\mathbf{t} A \mathbf{t}^{\dagger}-\boldsymbol{\nu} A \boldsymbol{\nu}^{\dagger}\right)
$$


Using this we can derive

$$
2\left(\frac{\partial^{2}}{\partial \theta^{2}}\left(\sqrt{\boldsymbol{\nu} A \boldsymbol{\nu}^{\dagger}}\right)+\sqrt{\boldsymbol{\nu} A \boldsymbol{\nu}^{\dagger}}\right)=2 \frac{\mathbf{t} A \mathbf{t}^{\dagger}}{\sqrt{\boldsymbol{\nu} A \boldsymbol{\nu}^{\dagger}}}-\frac{1}{2} \frac{\left(\mathbf{t} A \boldsymbol{\nu}^{\dagger}+\boldsymbol{\nu} A \mathbf{t}^{\dagger}\right)^{2}}{\left(\boldsymbol{\nu} A \boldsymbol{\nu}^{\dagger}\right)^{-3 / 2}},
$$

so that

$$
\mu_{1}(\tau, \boldsymbol{r})=\frac{\kappa}{\left[\left[u_{\mathrm{o}}^{ \pm}\right]\right]}\left(\sigma+\frac{\partial^{2} \sigma}{\partial \theta^{2}}\right)
$$

where the surface tension $\sigma$ is defined as

$$
\sigma=2 \int_{u_{-}}^{u_{+}} \sqrt{\boldsymbol{\nu} A \boldsymbol{\nu}^{\dagger} G(v)} \mathrm{d} v
$$

At this point it is interesting to observe that the expression for the surface tension $\sigma$ allows at most two-fold symmetry. This is in contrast to previous theories, e.g. [15] etc., where it was assumed that the surface tension reflects the symmetry of the underlying crystal lattice and could in principle have higher symmetry. Our analysis shows that in fact higher symmetries of the surface tension enter only through mechanical effects, which is shown in Part II of this study.

There is a very interesting different approach to modeling anisotropic surface tension, which is due to [1], [2]. There, the authors start from a nonlocal discrete spin lattice model in order to derive a hierarchy of higher gradient phase field models. However, they are interested in nonconserved order parameters, so that their treatment generalizes the Allen-Cahn model but not the Cahn-Hilliard models, which is the focus of the current study. But this is a minor point compared to the different origins for anisotropic surface tensions in [1], [2] and in our results. In our model, anisotropic gradient coefficients are in general mainly due to mechanical stresses in the crystal lattices of the two phases and they reflect the symmetry of the undistorted lattices. In [1], [2] anisotropic gradient coefficients result by taking into account higher gradient terms in the Taylor expansion of the nonlocal interaction function $J$.

\subsection{Generalization to $3 D$}

The sharp interface limit implies in first and second order the conditions (3.48) and (3.61), respectively. The condition (3.48) yields the equilibrium concentrations according to the common tangent construction, which describes the jump conditions if the curvature of the interface is ignored. The condition (3.61) takes care of curvature and yields the corrections to the plane interface case. Up to now we considered exclusively a phase mixture in $2 \mathrm{D}$ with interfaces as $1 \mathrm{D}$ objects. While the common tangent construction is not influenced by this restriction, the condition 3.61) is. A generalization to the case of a phase mixture in $3 \mathrm{D}$ with interfaces as $2 \mathrm{D}$ surfaces can be carried out along similar lines. In this case the interfaces are described by two Gaussian parameters $U^{\Delta}$, $\Delta \in\{1,2\}$, and the function $r\left(\tau, U^{1}, U^{2}\right)$ gives the location of the surface points. The surface is equipped with a unit normal $\nu$, two tangent vectors $t_{\Delta}$, a metric, $g_{\Delta \Gamma}$, and a Gaussian curvature tensor, $b_{\Delta \Gamma}$. The mean curvature is defined by $\kappa_{M}=\frac{1}{2} g^{\Delta \Gamma} b_{\Delta \Gamma}$. All these quantities can be calculated from the surface function $\boldsymbol{r}\left(\tau, U^{1}, U^{2}\right)$.

The expression corresponding to 3.61 reads

$$
\left[\left[u_{\mathrm{o}}^{ \pm}\right]\right] \mu_{1}=2 \sigma \kappa_{M}+\left(-\frac{1}{2} \sigma^{\Delta}\right)_{; \Delta},
$$


where the semicolon indicates the covariant derivative, and with

$$
\sigma=2 \int_{u_{-}}^{u_{+}} \sqrt{\boldsymbol{\nu} A \boldsymbol{\nu}^{\dagger} G(v)} \mathrm{d} v, \quad \sigma_{\Delta}=2 \int_{u_{-}}^{u_{+}}\left(\boldsymbol{\nu} A \mathbf{t}_{\Delta}^{\dagger}+\mathbf{t}_{\Delta} A \boldsymbol{\nu}^{\dagger}\right) \sqrt{\frac{G(v)}{\nu A \nu^{\dagger}}} \mathrm{d} v .
$$

The interfacial speed is just as in the 2D case and given in [7].

\subsection{A remark on the Gibbs adsorbtion equation}

We conclude from $(3.78)_{1}$ that the surface tension depends on the interfacial normal vector and on the limiting values of the concentrations of the adjacent phases. Moreover, the surface tension depends also on the temperature and elastic strain. However, these dependencies will be discussed in Part II of this study.

The normal and concentration dependence of $\sigma$ is explicitly given by $3.78,1$ because the phase field study of this model relies on atomistic reasonings. Phase field models that rely on phenomenological reasoning need a different source of information on the dependencies of the surface tension. In this case, the strategy is the identification of the Gibbs adsorbtion equation, which allows likewise the calculation of the surface tension, at least in phase equilibrium. The background and strategy are carefully described in [22], where also a phase field model of a binary mixture is formulated. Despite the fact that mechanical effects are ignored here, the main basic difference to our approach is the following. We start from the assumption that the phase field is given by the concentration itself. In [22] additionally to the concentration a phase field is introduced, to take care of the possibility that there might be no one-to-one correspondence between the present phase and the present concentration.

\section{Jump conditions for a binary mixture according to classical thermodynamics}

The sharp interface limit from Section 3 reveals jump conditions at the interface between the two coexisting phases. Jump conditions, however, can also be obtained from classical thermodynamics that models the interfaces from the very beginning as singular surfaces [7]. Here, we consider classical thermodynamics of a binary disordered mixture that may consist of two coexisting phases $\alpha$ and $\beta$, and we ignore mechanical stress fields in the bulk. In this case the variables are the temperature $T$ and the partial mass densities $\rho^{A}$ and $\rho^{B}$ of the two constituents $A$ and $B$. The specific free energy density is given by a function of the type

$$
\psi=\hat{\psi}\left(T, \rho^{A}, \rho^{B}\right),
$$

and

$$
\mu^{A}=\frac{\partial \rho \hat{\psi}}{\partial \rho^{A}} \quad \text { and } \quad \mu^{B}=\frac{\partial \rho \hat{\psi}}{\partial \rho^{B}}
$$

are the chemical potentials of the constituents (see e.g. [24]). Here $\rho=\rho^{A}+\rho^{B}$ denotes the mass density of the mixture.

It is useful to change the variables $\rho_{A}, \rho_{B}$ according to

$$
\left(\rho^{A}, \rho^{B}\right) \rightarrow\left(\rho, c=\rho^{B} / \rho\right) .
$$


We write

$$
\hat{\psi}\left(T, \rho^{A}, \rho^{B}\right)=\tilde{\psi}(T, \rho, c)
$$

and

$$
p=\rho^{2} \frac{\partial \rho \tilde{\psi}}{\partial \rho}
$$

defines the pressure. Next we calculate the chemical potentials from the function $\tilde{\psi}$. We obtain

$$
\mu^{A}=\psi+\frac{p}{\rho}-c \tilde{\psi}^{\prime} \quad \text { and } \quad \mu^{B}=\psi+\frac{p}{\rho}+(1-c) \tilde{\psi}^{\prime} \quad \text { with } \quad \tilde{\psi}^{\prime}=\frac{\partial \tilde{\psi}}{\partial c} .
$$

Note that

$$
\mu \equiv \mu^{B}-\mu^{A}=\tilde{\psi}^{\prime} .
$$

The jump conditions at the interface are derived in classical thermodynamics by means of generic balance equations [7]. If we denote the limits of a generic quantity $g$ obtained by approaching the interface from the $\alpha$-phase and $\beta$-phase, respectively, by $g_{\alpha}$ and $g_{\beta}$, the jump conditions read

$$
\mu_{\beta}^{A}-\mu_{\alpha}^{A}=0, \quad \mu_{\beta}^{B}-\mu_{\alpha}^{B}=0, \quad p_{\beta}-p_{\alpha}=S^{\Delta \Gamma} b_{\Delta \Gamma}+S_{; \Delta}^{\Delta} .
$$

The newly introduced quantities are the tangential surface stress, $S^{\Delta \Gamma}$, the normal surface stress, $S^{\Delta}$, and the semicolon indicates the covariant derivative.

Thermodynamics of interfaces relates the surface stresses $S^{\Delta \Gamma}$ and $S^{\Delta}$ to the free energy density, $\psi_{s}$, of the interface. Under the assumptions that

(i) $T_{\beta}=T_{\alpha} \equiv T$,

(ii) $\psi_{s}$ may depend on $T, v^{i}$ and $g_{\Delta \Gamma}$,

one obtains as a consequence of the second law of thermodynamics

$$
S^{\Delta \Gamma}=\psi_{s} g^{\Delta \Gamma}+\frac{1}{2} \frac{\partial \psi_{s}}{\partial g_{\Delta \Gamma}} \quad \text { and } \quad S^{\Delta}=-g^{\Delta \Gamma} \tau_{\Gamma}^{i} \frac{\partial \psi_{s}}{\partial \nu^{i}}
$$

(see [7] for details). The first contribution of $S^{\Delta \Gamma}$ leads in 4.8$]_{3}$ to the classical capillary force which is proportional to the mean curvature $\kappa_{M}=\frac{1}{2} g^{\Delta \Gamma} b_{\Delta \Gamma}$. The metric dependence of the interfacial free energy describes elastic effects of the interface and the normal surface stress, given by $(4.9)_{2}$, which is related to the Cahn-Hoffmann vector.

Next we will evaluate the jump condition (4.8). First we write the conditions $(4.8)_{1,2}$ more explicitly:

$$
\tilde{\psi}_{\beta}^{\prime}\left(T, \rho_{\beta}, c_{\beta}\right)=\tilde{\psi}_{\alpha}^{\prime}\left(T, \rho_{\alpha}, c_{\alpha}\right) \equiv \mu \quad \text { and } \quad\left(c_{\beta}-c_{\alpha}\right) \mu=\tilde{\psi}_{\beta}-\tilde{\psi}_{\alpha}+\frac{p_{\beta}}{\rho_{\beta}}-\frac{p_{\alpha}}{\rho_{\alpha}} .
$$

Let us assume for simplicity $\rho_{\beta} \approx \rho_{\alpha} \equiv \rho$, and let $c_{\beta_{0}}, c_{\alpha_{0}}$ be the solution of

$$
\tilde{\psi}_{\beta}^{\prime}\left(T, \rho, c_{\beta}\right)=\tilde{\psi}_{\alpha}^{\prime}\left(T, \rho, c_{\alpha}\right) \quad \text { and } \quad\left(c_{\beta}-c_{\alpha}\right) \mu=\tilde{\psi}_{\beta}-\tilde{\psi}_{\alpha},
$$

which describes the common tangent construction, also called the Maxwell construction. We conclude that the common tangent construction only holds if

(i) $p_{\beta}=p_{\alpha} \equiv p_{0}$,

(ii) $\rho_{\beta}=\rho_{\alpha} \equiv \rho_{0}$. 
Note at this point that condition (i) is well known, but the necessity of the second condition is in general not noted. In the following we will take the condition (ii) for granted. Note that in the derivation of the sharp-interface limit from the reduced phase field model we also did not consider the variation of the total density of the binary mixture. Furthermore, note that this variation is related to the trace of the mechanical strain, which will be included in Part II of this study.

We proceed to exploit the jump conditions 4.8 . To this end we make the Ansatz

$$
c_{\alpha}=c_{\alpha_{0}}+c_{\alpha_{1}}, \quad c_{\beta}=c_{\beta_{0}}+c_{\beta_{1}}, \quad p_{\alpha}=p_{\alpha_{0}}+p_{\alpha_{1}}, \quad p_{\beta}=p_{\beta_{0}}+p_{\beta_{1}}, \quad \mu=\mu_{0}+\mu_{1}
$$

and exploit the jump condition $(4.10]_{1}$ under the assumption that quantities with index 1 are small corrections to the corresponding quantities with index 0 . We obtain

$$
\mu_{0}=\tilde{\psi}_{\alpha}^{\prime}\left(T, \rho_{0}, c_{\alpha_{0}}\right)=\tilde{\psi}_{\beta}^{\prime}\left(T, \rho_{0}, c_{\beta_{0}}\right), \quad \mu_{1}=\tilde{\psi}_{\alpha}^{\prime \prime}\left(T, \rho_{0}, c_{\alpha_{0}}\right) c_{\alpha_{1}}=\tilde{\psi}_{\beta}^{\prime \prime}\left(T, \rho_{0}, c_{\beta_{0}}\right) c_{\beta_{1}},
$$

while the conditions 4.10$]_{2}$ and 4.8$]_{2}$ imply

$$
\left(c_{\beta_{0}}-c_{\alpha_{0}}\right) \mu_{1}=\frac{1}{\rho_{0}}\left(S^{\Delta \Gamma} b_{\Delta \Gamma}+S_{; \Delta}^{\Delta}\right) .
$$

Let us now ignore elastic effects of the interface so that the right hand side of (4.14) can be rewritten as

$$
\left(c_{\beta_{0}}-c_{\alpha_{0}}\right) \mu_{1}=\frac{1}{\rho_{0}}\left(2 \psi_{s} \kappa_{M}+\left(-g^{\Delta \Gamma} \tau_{\Gamma}^{i} \frac{\partial \psi_{s}}{\partial \nu^{i}}\right)_{; \Delta}\right) .
$$

A comparison of 4.15) with the corresponding result 3.77), which we have obtained from the phase field model, suggests identifying

$$
\psi_{s}=\sigma .
$$

In this case the definitions 3.78 imply that the interfacial free energy density has the following properties:

$$
v^{i} \frac{\partial \psi_{s}}{\partial \nu^{i}}=\psi_{s} \quad \text { and } \quad \tau_{\Delta}^{i} \frac{\partial \psi_{s}}{\partial \nu^{i}}=\frac{1}{2} \sigma_{\Delta} .
$$

The result 4.15) of classical thermodynamics can thus be written

$$
\rho_{0}\left(c_{\beta_{0}}-c_{\alpha_{0}}\right) \mu_{1}=2 \sigma \kappa_{M}+\left(-\frac{1}{2} \sigma^{\Delta}\right)_{\Delta} .
$$

This result is in agreement with (3.77) developed from the phase field model.

Originally, the Cahn-Hoffmann vector, $\xi^{i}$, was introduced to describe interfaces with anisotropic surface tension within sharp-interface models. It was shown, e.g. in [21], that the Cahn-Hoffmann vector can also be identified with the phase field models and they provide useful generalizations of the classical concepts. In the current study we can also identify the CahnHoffmann vector, which is here related to the normal surface stress by $S^{\triangle}=-g^{\Delta \Gamma} \tau_{\Gamma}^{K} \xi^{K}$ (see also [7] for details). A comparison with 4.17 and $4.92_{2}$ yields thus the identification $\tau_{\Gamma}^{K} \xi^{K}=\frac{1}{2} \sigma_{\Gamma}$, which gives the tangential component of the Cahn-Hoffmann vector within the phase field model, via equation $3.78 p_{2}$. However, the role of $\xi$ changes, if we consider the elastic contributions of the current model to the gradient coefficients. A detailed discussion of this topic is found in Part II of this study. 


\section{Numerical methods}

For our numerical treatment we follow [15] and transform the sharp-interface model $(3.63)-(3.66$, (3.71) into a boundary integral formulation. Note that the sharp-interface model describes mass diffusion in the matrix and mass diffusion in the precipitates connected by the common boundary condition 3.65. Here, we make the simplifying assumption that diffusion in the precipitates can be neglected, i.e. we treat only the one-sided model

$$
\begin{aligned}
\Delta \mu_{1}^{-} & =0 & & \text { in } \Omega_{-}, \\
\boldsymbol{n} \cdot \nabla \mu_{1}^{-} & =0 & & \text { on } \partial \Omega_{-}, \\
\mu_{1}^{-} & =\tilde{\mu}_{1} & & \text { on } \Gamma, \\
{\left[\left[u_{\mathrm{o}}^{ \pm}\right]\right] V^{\nu} } & =\boldsymbol{\nu} \cdot \nabla \mu_{1}^{-} & & \text {on } \Gamma,
\end{aligned}
$$

and drop the superscript $(-)$ from now on. Note that this does restrict the range of morphological scenarios in comparison to the solutions of the phase field model (see [19]). However, we expect the shapes shown here to qualitatively correspond to those of a two-sided model for very small diffusion inside the precipitate.

We further assume that $\partial \Omega$ has been shifted to infinity and replace the local condition $\boldsymbol{n} \cdot \boldsymbol{\nabla} \mu_{1}=0$ by the condition of no flux in the far field

$$
\lim _{R_{\infty} \rightarrow \infty} \int n \cdot \nabla \mu_{1}=0 .
$$

In what follows, we note that the tangential vector $\boldsymbol{t}$ to the boundary of a precipitate always points into the mathematically positive direction, and $\nu$ always points to the left, i.e. inside the precipitate.

We next derive a boundary integral representation for the solution of $\Delta \mu_{1}=0$ in $\Omega_{-}$by setting

$$
\mu_{1}=\operatorname{Re}(\Psi(z)), \quad z=x+i y, z \in \Omega_{-}
$$

where

$$
\Psi(z)=\sum_{k=1}^{N} A_{k} \ln \left(z-M_{k}\right)+\frac{1}{2 \pi i} \int_{\Gamma} \frac{\Phi(\zeta)}{\zeta-z} d \zeta+\frac{1}{2 \pi} \int \Phi(\zeta) \mathrm{d} \zeta
$$

Here $A_{k} \in \mathbb{R}, \Phi$ is a complex function and the complex number $M_{k}$ is in $\Omega_{k}$, the interior of the $k$-th precipitate, bounded by $\Gamma_{k}$, where $\Gamma:=\bigcup_{k=1}^{N} \Gamma_{k}$. The first term on the r.h.s. represents the contribution of the 2D-precipitates, the second term is the analytical part for $z \in \Omega_{-}$and the third part is a constant correction if $\Psi \not \rightarrow 0$ as $z \rightarrow \infty$. As shown by Mikhlin [23] this representation is unique for given $z_{k}$. Furthermore, it is shown in [23] that for $z \rightarrow \tilde{z} \in \Gamma$,

$$
\begin{aligned}
\operatorname{Re}\left(\lim _{\substack{z \rightarrow \tilde{z} \\
z \in \Omega_{-}}} \Psi\right) & =-\frac{1}{2} \Phi(\tilde{z})+\sum_{k=1}^{N} A_{k} \ln \left(\tilde{z}-M_{k}\right)+\frac{1}{2 \pi} \operatorname{Im} \int_{\Gamma} \frac{\Phi(\zeta)}{\zeta-z} \mathrm{~d} \zeta+\frac{1}{2 \pi} \int \Phi(\zeta) \mathrm{d} \zeta \\
& =\left.\mu_{1}\right|_{\Gamma}
\end{aligned}
$$

i.e. for $\mu_{1}$ on $\Gamma$. Substitution of (5.6), (5.7) into (5.5) yields

$$
\sum_{k=1}^{N} A_{k}=0
$$


Note that the 1.h.s. of (5.8) is an integral operator on $\Phi$ with kernel of dimension $N-1$ so that we have $N-1$ solvability conditions for the r.h.s. of (5.8). Together with 5.9p this will fix the $A_{k}$, so that we can find the corresponding $\Phi$. In order to obtain uniqueness one has to impose the constraints (see again [23])

$$
\int_{\Gamma_{k}} \Phi(\zeta) \mathrm{d} \zeta=0
$$

Now we can determine $\mu_{1}$ on $\Gamma$ and hence $\nabla \mu_{1}$, which allows us to calculate $\left[\left[u_{\mathrm{o}}^{ \pm}\right]\right] V^{\boldsymbol{\nu}}=\boldsymbol{\nu} \cdot \boldsymbol{\nabla} \mu_{1}$. Note that knowledge of the normal velocity is sufficient to evolve the interface. For the numerical implementation we use a parametrization $z_{k}(\alpha, \tau)$ for each $\Gamma_{k}$, where $\alpha \in[0,2 \pi]$. These parameter functions are evolved according to

$$
\frac{\mathrm{d} z_{k}}{\mathrm{~d} \tau}=V_{k}^{\nu} \nu_{k}+V_{k}^{\mathbf{t}} \boldsymbol{t}_{k}
$$

where $\boldsymbol{\nu}_{k}$ and $\boldsymbol{t}_{k}$ denote the normal and tangent vectors with respect to the $k$-th precipitate. The tangential component $V_{k}^{\mathbf{t}}$ of $\mathrm{d} z_{k} / \mathrm{d} \tau$ remains arbitrary and a special choice of the parametrization for the boundaries $\Gamma_{k}$ will be used to simplify the numerical implementation. Here we follow [16] in the choice of the coordinate system, where $\theta_{k}$ is the angle of the tangent vector at points on $\Gamma_{k}$ to the $x$-axis and $L_{k}$ denotes the length of the corresponding interface. The components of $z_{k}(\alpha, \tau)$ are then replaced by the coordinates $s_{k \alpha}$ and $\theta_{k}$ through

$$
s_{k \alpha}=\left|z_{k}\right| \quad \text { and } \quad\left(\cos \theta_{k}(\alpha, \tau), \sin \theta_{k}(\alpha, \tau)\right)=\frac{\left(\operatorname{Re}\left(\boldsymbol{z}_{k}\right), \operatorname{Im}\left(\boldsymbol{z}_{k}\right)\right)}{s_{k \alpha}}
$$

so that the evolution equations

$$
\frac{\partial s_{k \alpha}}{\partial \tau}=V_{k \alpha}^{\mathbf{t}}-\theta_{k \alpha} V_{k}^{\nu} \quad \text { and } \quad \frac{\partial \theta_{k}}{\partial \tau}=\frac{V_{k \alpha}^{\nu}+V_{k}^{\mathbf{t}} \theta_{k \alpha}}{s_{k \alpha}}
$$

are obtained. Note that the index $k$ refers to the $k$-th precipitate, while the index $\alpha$ denotes partial derivative with respect to the parameter $\alpha$. Then the special choice of

$$
V_{k}^{\mathbf{t}}(\alpha, \tau)=\int_{0}^{\alpha} \theta_{k \alpha^{\prime}} V_{k}^{\nu} \mathrm{d} \alpha^{\prime}-\frac{\alpha}{2 \pi} \int_{0}^{2 \pi} \theta_{k \alpha^{\prime}} V_{k}^{\nu} \mathrm{d} \alpha^{\prime}
$$

yields the equal arclength parametrization

$$
s_{k \alpha}=\frac{L_{k}(\tau)}{2 \pi} \quad \text { for all } \alpha,
$$

and hence the simpler ODE-PDE system

$$
\frac{\partial L_{k}}{\partial \tau}=-\int_{0}^{2 \pi} \theta_{k \alpha^{\prime}} V_{k}^{\nu} \mathrm{d} \alpha^{\prime} \quad \text { and } \quad \frac{\partial \theta_{k}}{\partial \tau}=\frac{2 \pi}{L_{k}}\left(V_{k \alpha}^{\nu}+\theta_{k \alpha} V_{k}^{\mathbf{t}}\right) .
$$


In summary, the complete boundary integral formulation for the evolution of the precipitates is:

$$
\begin{gathered}
-\frac{1}{2} \Phi_{k}(\alpha, \tau)+\frac{1}{2 \pi} \sum_{l=1}^{N} \int_{0}^{2 \pi} \Phi_{l}\left(\alpha^{\prime}, \tau\right) \operatorname{Im}\left(\frac{z_{l \alpha^{\prime}}\left(\alpha^{\prime}\right)}{z_{l}\left(\alpha^{\prime}\right)-z_{l}(\alpha)}\right) \mathrm{d} \alpha^{\prime}+\frac{1}{L_{k}} \sum_{l=1}^{N} \int_{0}^{2 \pi} \Phi_{l}\left(\alpha^{\prime}, \tau\right) \mathrm{d} \alpha^{\prime} \\
\quad+\sum_{l=1}^{N} A_{l} \ln \left(z_{l}(\alpha)-M_{l}\right)=\mu_{1} \mid \Gamma \\
\int_{0}^{2 \pi} \Phi_{k}\left(\alpha^{\prime}, \tau\right) \mathrm{d} \alpha^{\prime}=0 \quad k=1, \ldots, N-1 \\
V_{k}^{\nu}(\alpha, \tau)=-\frac{1}{L_{k}} \sum_{l=1}^{N} \int_{0}^{2 \pi} \Phi_{l \alpha^{\prime}}\left(\alpha^{\prime}, \tau\right) \operatorname{Re}\left(\frac{z_{l \alpha}(\alpha)}{z_{l}\left(\alpha^{\prime}\right)-z_{l}(\alpha)}\right) \mathrm{d} \alpha^{\prime}
\end{gathered}
$$

together with (5.9), 5.14) and 5.16).

Now, consider $\theta_{k \alpha}$ in the second equation of 5.16. It gets another derivative in the first equation of 5.16) and another one in 5.17), since in $\theta, s_{\alpha}$ coordinates the curvature in $\left.\mu_{1}\right|_{\Gamma}$ is expressed as $\kappa=\theta_{\alpha} / s_{\alpha}$. Hence, there are three derivatives. Such high derivatives in an evolution equation will lead to numerically stiff problems (the stability constraint e.g. $\Delta t<O\left((\triangle x)^{3}\right)$ leads to prohibitive time stepping). However, the advantage of the above formulation is that the evolution equation can for each $k$ be written as

$$
\frac{\partial \theta}{\partial \tau}=\left(\frac{2 \pi}{L}\right)^{3} \bar{\mu} \mathcal{H}\left[\theta_{\alpha \alpha \alpha}\right]+N(\alpha, \tau),
$$

where

$$
\bar{\mu}=\frac{1}{\left[u_{ \pm}\right]} \int_{0}^{2 \pi} \sigma \mathrm{d} \theta .
$$

The first term of 5.20 becomes in Fourier space

$$
-\left(\frac{2 \pi}{L}\right)^{3}|j|^{3} \theta
$$

which is the stiffest term and will be treated implicitly. However, in this form it is linear and diagonal in Fourier space and hence one only has to solve a diagonal system. The remaining complicated nonlinear expression $N(\alpha, \tau)$ can be treated explicitly. We use a pseudo-spectral method (using FFT) in space, and Leap-Frog for the explicit and Crank-Nicholson for the implicit time integration. The integration of the $L_{\tau}$ ODE is done with an Adams-Bashforth integrator. Equations (5.17), (5.18), 5.19) and 5.9) yield the $\Psi$ and $A_{k}$. They represent a linear system which we solve iteratively using GMRES.

\section{$5.1 \quad$ Examples}

We are interested here in some first characteristic features of the influence of anisotropic surface tension on the dynamics of coarsening. This will be extended to include elastic effects in Part II.

For the numerical simulations we use for $A(\tilde{u})$ the linear interpolating expression

$$
A(\tilde{u})=\left(\begin{array}{cc}
a^{\alpha} & 0 \\
0 & a^{\alpha}
\end{array}\right) \tilde{u}+\left(\begin{array}{cc}
a_{1}^{\beta} & 0 \\
0 & a_{2}^{\beta}
\end{array}\right)(1-\tilde{u}),
$$


where the $\alpha$-matrix corresponds to a cubic symmetry and the $\beta$-matrix to a tetragonal symmetry, frequently occurring in binary alloys (see e.g. [9]). For the configurational part of the free energy we choose a fourth order polynomial, i.e. $F^{\prime}\left(\tilde{u}_{\mathrm{o}}\right)=\tilde{u}_{\mathrm{o}}\left(\tilde{u}_{\mathrm{o}}-1 / 2\right)\left(\tilde{u}_{\mathrm{o}}-1\right)$ so that $b_{0}=0$, $\left[\left[u_{\mathrm{o}}^{ \pm}\right]\right]=1$ and $G(v)=1 / 4 v^{2}(v-1)^{2}$. With this choice we obtain the following explicit expression for $\sigma$ :

where

$$
\sigma=\sqrt{a^{\alpha}} \frac{4}{105 p^{3}}\left[(1-p)^{3 / 2}\left(4-p-3 p^{2}\right)-4+7 p\right],
$$

$$
p=q+\delta \sin ^{2} \theta \quad \text { with } \quad q=\frac{a^{\alpha}-a_{1}^{\beta}}{a^{\alpha}}, \quad \delta=\frac{a_{2}^{\beta}-a_{1}^{\beta}}{a^{\alpha}} .
$$

In all simulations for Figures $5-77$ we let $q=0.4$ and $\delta=0.1$. For the initial conditions we always choose a pair of circles, where the left one has its center at the origin and is of radius $R=0.13$ and the right one has its center shifted to the right by $d>2.01 R$ from the origin and has $1 \%$ larger radius. We are interested in the effect of anisotropic surface tension and distance of the precipitates on the coarsening rate and their shape.
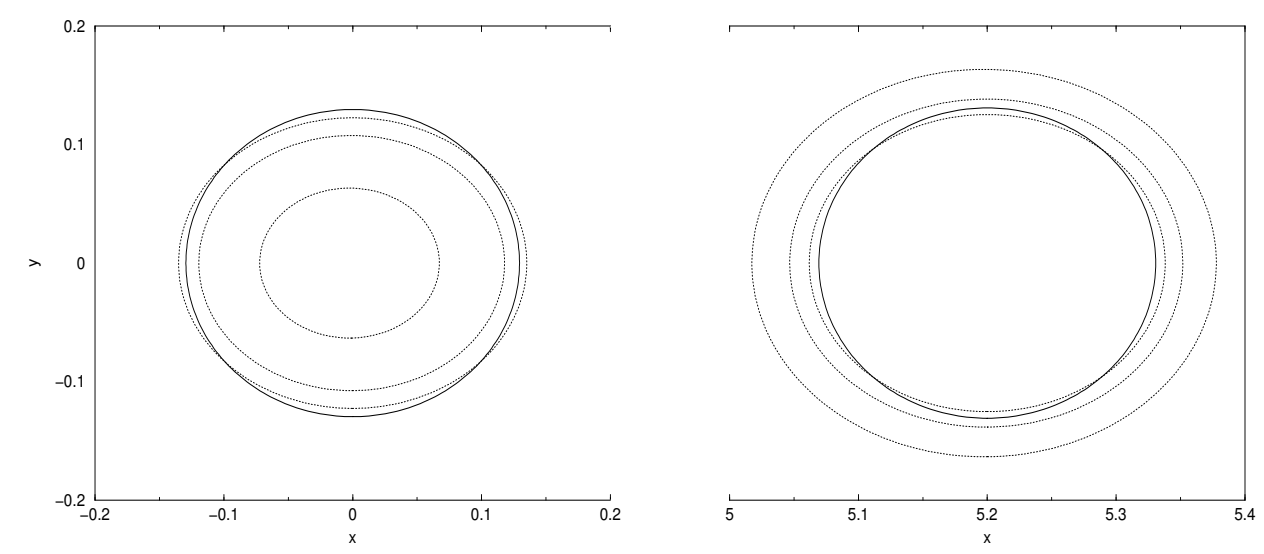

FIG. 5. Two evolving precipitates with centers initially $d=5.2$ apart (solid line). Middle portion $0.2<x<5.2$ not shown.

In Figure 5 we see the change in area for two precipitates, initially of circular shape (solid line), where the second one has center shifted by $d=5.2$ to the right and having $1 \%$ larger radius (also solid line). Note that we do not show the middle portion $0.2<x<5$ to better focus on the shape of the precipitates. For both precipitates, the growing (right) and the shrinking (left) one, coarsening proceeds by quickly assuming and retaining almost equilibrium shape (dotted lines), which can be found analytically as the stationary solution of (5.17)-(5.19), (5.9), (5.14) and 5.16), for a single precipitate

$$
\begin{aligned}
& r_{1 \text { stat }}=\int_{0}^{s} \cos \theta \mathrm{d} s=\int_{0}^{\theta} \frac{\cos \theta}{\partial \theta / \partial s} \mathrm{~d} \theta=-\frac{1}{C}\left(\sigma \sin \theta+\frac{\partial \sigma}{\partial \theta} \cos \theta\right), \\
& r_{2 \text { stat }}=\int_{0}^{s} \sin \theta \mathrm{d} s=\int_{0}^{\theta} \frac{\sin \theta}{\partial \theta / \partial s} \mathrm{~d} \theta=\frac{1}{C}\left(\sigma \cos \theta-\frac{\partial \sigma}{\partial \theta} \sin \theta\right)
\end{aligned}
$$

with scaling factor $C$. 


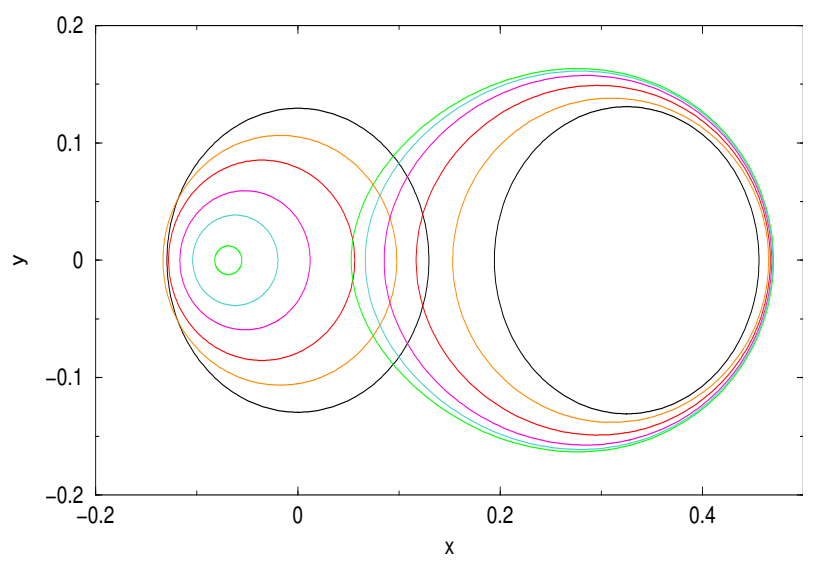

FIG. 6. Two evolving precipitates with centers initially $d=0.325$ apart.

In Figure 6 the center of the left initial circle is the origin, while the center of the right initial circle has been shifted only by $d=0.325$ to the right. We see that while the right precipitate is growing at the expense of the left one, the influence of diffusion dominates the shape of the precipitates. Only for the smallest precipitate shown is the equilibrium shape attained, i.e. when surface tension dominates.

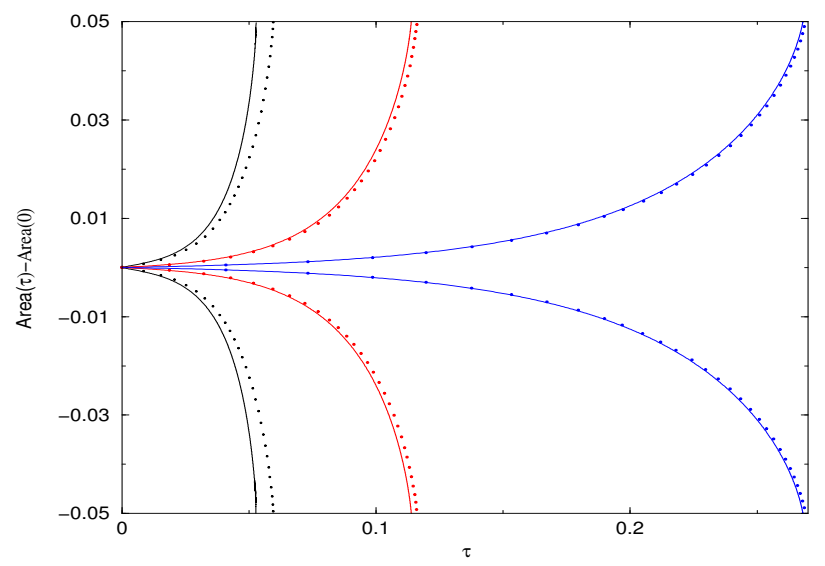

FIG. 7. Change in area for two precipitates, placed next to each other (solid line) and over each other (dotted line).

In Figure 7 we show the influence of distance of the precipitates on the coarsening rate, which is smaller for larger distances. Additionally, we performed a second set of simulations, where the center of the second precipitate was placed a distance $d$ above the origin instead to the right. While the influence of the distance on the shape is analogous to the previous examples, we also notice a dependence of the coarsening rate on the orientation of the precipitates with respect to each other. Figure 7 shows the area of the precipitates minus their initial area for three pairs initially with 
distance $d=0.325$ (left), $d=0.65$ (middle) and $d=5.2$ (right). The solid lines represent pairs of precipitates, where the center of the second one is placed to the right of the origin, as in the previous figures, and the dotted lines represent those where the center of the second precipitate is placed above the origin. We see that the effect of anisotropic surface tension on the coarsening is felt largely for nearby particles. Furthermore, we see a tendency that coarsening proceeds faster when the points of higher curvature are closer.

\section{Conclusion}

In this paper we derived a phase field model for a binary alloy such as $S n P b$ from an atomistic point of view. The resulting model has anisotropic surface energy with a nonlinear dependence on concentration. For the study of phase separation we employ the method of matched asymptotic expansion to obtain a corresponding sharp-interface model for the case when mechanical effects are neglected. As a result we also obtain an analytic expression for the concentration dependent surface tension. Interestingly, the expression allows at most a two-fold symmetry. It furthermore agrees with the one obtained on the basis of classical thermodynamics for jump conditions at singular interfaces.

We finally investigate the dynamics of precipitates numerically, using a boundary integral formulation of our sharp-interface model.

\section{Acknowledgements}

We would like to thank Harald Garcke for many stimulating discussions. BW would also like to thank Andreas Münch for all his help with the numerical part of this paper.

\section{Appendix A. Higher gradient coefficients}

In this appendix we relate the coefficients which appear in $\sqrt{2.13}$ to the three pair potentials $\varphi_{\gamma}^{A A}$, $\varphi_{\gamma}^{B B}, \varphi_{\gamma}^{A B}$ and to the combinations 2.17, respectively. Recall the definitions $\Delta_{i}^{a b}=X_{i}^{b}-X_{i}^{a}$, $\Delta^{a b}=\left|X_{i}^{b}-X_{i}^{a}\right|$ and let us furthermore set $N_{i}=\Delta_{i}^{a b} / \Delta^{a b}$.

The coefficients determining the local part of the free energy read

$$
\psi_{\gamma}^{A A}=\frac{1}{2} \sum_{b} \varphi_{\gamma}^{A A}\left(\Delta^{a b}\right), \quad \psi_{\gamma}^{\tilde{\varphi}}=\frac{1}{2} \sum_{b} \tilde{\varphi}_{\gamma}\left(\Delta^{a b}\right), \quad \psi_{\gamma}^{\varphi}=\frac{1}{2} \sum_{b} \varphi_{\gamma}\left(\Delta^{a b}\right) .
$$

The higher gradient coefficients, which we also call extended Cahn-Hilliard coefficients, read

$$
a_{\gamma \mid k l}^{\varphi}=\frac{1}{2} \sum_{b}\left(\Delta^{a b}\right)^{2} \varphi_{\gamma}\left(\Delta^{a b}\right) N_{k} N_{l}, \quad a_{\gamma \mid k l}^{\tilde{\varphi}}=\frac{1}{2} \sum_{b}\left(\Delta^{a b}\right)^{2} \tilde{\varphi}_{\gamma}\left(\Delta^{a b}\right) N_{k} N_{l},
$$

and

$$
\begin{aligned}
& A_{\gamma \mid k l m n o p}^{\varphi}=\frac{1}{2} \sum_{b}\left(\Delta^{a b}\right)^{4} \frac{\partial^{2} \varphi_{\gamma}\left(\Delta^{a b}\right)}{\partial \Delta_{m}^{a b} \partial \Delta_{o}^{a b}} N_{k} N_{l} N_{n} N_{p}, \\
& A_{\gamma \mid k \text { lmnop }}^{\tilde{\varphi}}=\frac{1}{2} \sum_{b}\left(\Delta^{a b}\right)^{4} \frac{\partial^{2} \tilde{\varphi}_{\gamma}\left(\Delta^{a b}\right)}{\partial \Delta_{m}^{a b} \partial \Delta_{o}^{a b}} N_{k} N_{l} N_{n} N_{p} .
\end{aligned}
$$


Finally, there are the coefficients which determine the elastic stiffness matrix, and these are

$$
\begin{aligned}
& A_{\gamma \mid \text { mnop }}^{A A}=\sum_{b}\left(\Delta^{a b}\right)^{2} \frac{\partial^{2} \varphi_{\gamma}^{A A}\left(\Delta^{a b}\right)}{\partial \Delta_{m}^{a b} \partial \Delta_{o}^{a b}} N_{k} N_{l}, \quad A_{\gamma \mid \text { mnop }}^{\varphi}=\sum_{b}\left(\Delta^{a b}\right)^{2} \frac{\partial^{2} \varphi_{\gamma}\left(\Delta^{a b}\right)}{\partial \Delta_{m}^{a b} \partial \Delta_{o}^{a b}} N_{k} N_{l}, \\
& A_{\gamma \mid \text { mnop }}^{\tilde{\varphi}}=\sum_{b}\left(\Delta^{a b}\right)^{2} \frac{\partial^{2} \tilde{\varphi}_{\gamma}\left(\Delta^{a b}\right)}{\partial \Delta_{m}^{a b} \partial \Delta_{o}^{a b}} N_{k} N_{l} .
\end{aligned}
$$

We conclude that the constitutive law for the phase field model is completely determined by the three pair potential functions. How these functions can be fitted to experimental data is described in [10].

\section{REFERENCES}

1. Caginalp, G. The role of microscopic anisotropy in the macroscopic behavior of a phase boundary. Ann. Phys. 172 (1986), 136-155. Zbl 0639.58038 MR 0912765

2. Caginalp, G. \& Fife, P. Higher order phase-field models and detailed anisotropy. Phys. Rev. B 34 (1986), 4940-4943. MR 0875362

3. CAhn, J. W. Thermodynamics of solid and fluid surfaces. Interfacial Segregation, W. C. Johnson and J. M. Blakely (eds.), Amer. Soc. of Metals, Metals Park, OH (1978), 3-23.

4. CAhn, J. W. \& Hilliard, J. E. Free energy of a nonuniform system. I. Interfacial free energy. J. Chem. Phys. 28 (1958), 258-267.

5. CAhn, J. W. \& Hoffmann, D. W. A vector thermodynamics for anisotropic surfaces II: Curved and faceted surfaces. Acta Met. 22 (1974), 1205-1214.

6. CAHN, J. W. \& NOVICK-COHEN, A. Evolution equations for phase separation and ordering in binary alloys. J. Statist. Phys. 76 (1994), 877-909. Zbl 0840.35110

7. Dreyer, W. On jump conditions at phase boundaries. WIAS preprint No. 869, 2003.

8. Dreyer, W. \& Kunik, M. Cold, thermal and oscillator closure of the atomic chain. J. Phys. A: Math. Gen. 33 (2000), 2097-2129. Zbl 0969.82027 MR 1748742

9. Dreyer, W. \& Müller, W. H. A study of the coarsening in tin/lead solders. Int. J. Solids Struct. 37 (2000), 3841-3871. Zbl 0973.74059

10. Dreyer, W. \& Müller, W. H. Modeling diffusional coarsening in eutectic tin/lead solders: A quantitative approach. Int. J. Solids Struct. 38 (2001), 1433-1458. Zbl 0961.74523

11. Foltyn, T. The effect of coarsening on near eutectic lead-tin alloy. MSc thesis, Department of Mechanical and Chemical Engineering, Heriot-Watt Univ. (2000).

12. DE Fontaine, D. Clustering effect in solid solutions. Treatise on Solid State Chemistry, N. B. Hannah (ed.), Plenum, New York (1975), 129-178.

13. Garcke, H., Nestler, B., \& Stoth, B. On anisotropic order parameter models for multiphase systems and their sharp interface limits. Phys. D 115 (1998), 87-108. Zbl 0936.82010 MR 1616772

14. Hoffmann, D. W. \& CAhn, J. W. A vector thermodynamics for anisotropic surfaces I: Fundamentals and applications to plane surface junctions. Surface Sci. 31 (1972), 368-388.

15. Hou, T. J., Leo, P. H., \& Lowengrub, J. S. Microstructural evolution in inhomogeneous elastic media. J. Comput. Phys. 131 (1997), 109-148.

16. Hou, T. J., Lowengrub, J. S., \& Shelley, M. J. Removing the stiffness from interfacial flows with surface tension. J. Comput. Phys. 114 (1994), 312-338. Zbl 0810.76095 MR 1294935

17. LAU, J. H. \& RiCE, D. W. Solder joint fatigue in surface mount technology: State of the art. Solid State Technology, (1985), 91-104. 
18. LEIBFrIED, G. Gittertheorie der mechanischen und thermischen Eigenschaften der Kristalle. Encyclopedia of Physics, Vol. VII, Part I, Crystal Physics I, Springer (1955).

19. Leo, P. H., Lowengrub, J. S., \& Jou, H. J. A diffuse interface model for microstructural evolution in elastically stressed solids. Acta Mater. 46 (1998), 2113-2130.

20. Leo, P. H., Lowengrub, J. S., \& Nie, Q. Microstructural evolution in orthotropic elastic media. J. Comput. Phys. 157 (2000), 44-88. Zbl 0960.74076 MR 1736884

21. McFadden, G. B. \& Wheeler, A. A. On the notion of a $\xi$-vector and a stress tensor for a general class of anisotropic diffuse interface models. Proc. Roy. Soc. London Sect. A 453 (1997), 1611-1630. Zbl 0894.60101 MR 1469165

22. McFadden, G. B. \& Wheeler, A. A. On the Gibbs adsorption equation and diffuse interface models. Proc. Roy. Soc. London Sect. A 458 (2002), 1129-1149. Zbl 0996.82023 MR 1902579

23. Mikhlin, S. G. Integral Equations and Their Applications to Certain Problems in Mechanics, Mathematical Physics, and Technology. Pergamon, New York (1957). Zbl 0077.09903 MR 0087877

24. MÜLler, I. Thermodynamics. Interaction of Mechanics and Mathematics, Pitman Adv. Publ. Program (1985). Zbl 0637.73002

25. Pego, R. L. Front migration in the nonlinear Cahn-Hilliard equation. Proc. Roy. Soc. London Sect. A 422 (1989), 261-278. Zbl 0701.35159 MR 0997638

26. Steinbach, I., Pezzolla, F., Nestler, B., Seesselberg, M., Prieler, R., Schmitz, G. J., \& Rezende, J. L. L. A phase-field concept for multiphase systems. Phys. D 94 (1996), 135-147. Zbl 0885.35148

27. Vianco, P. T., Burchett, S. N., Neilsen, M. K, Rejent, J. A., \& Frear, D. R. Coarsening of the $\mathrm{Sn} P b$ solder microstructure in constitutive model-based predictions of soder joint thermal mechanical fatigue. J. Electronic Materials 28 (1999), 1290-1298.

28. Wheeler, A. A., McFadden, G. B., \& Boettinger, W. J. Phase-field model for solidification of a eutectic alloy. Proc. Roy. Soc. London Sect. A 452 (1996), 495-525. 